- Original Paper •

\title{
Comparison of a Manual and an Automated Tracking Method for Tibetan Plateau Vortices
}

\author{
Julia CURIO ${ }^{* 1,2}$, Yongren CHEN $^{3,4}$, Reinhard SCHIEMANN ${ }^{1,2}$, Andrew G. TURNER ${ }^{1,2}$, \\ Kai Chi WONG ${ }^{1,2}$, Kevin HODGES ${ }^{2}$, and Yueqing $\mathrm{LI}^{3,4}$ \\ ${ }^{1}$ NCAS-Climate, University of Reading, Reading, RG6 6BB, UK \\ ${ }^{2}$ Department of Meteorology, University of Reading, Reading, RG6 6BB, UK \\ ${ }^{3}$ Institute of Plateau Meteorology, China Meteorological Administration, Chengdu, 610072, China \\ ${ }^{4}$ Heavy Rain and Drought-Flood Disasters in Plateau and Basin Key Laboratory of Sichuan Province, Chengdu, 610072, China
}

(Received 1 November 2017; revised 16 March 2018; accepted 21 March 2018)

\begin{abstract}
Tibetan Plateau vortices (TPVs) are mesoscale cyclones originating over the Tibetan Plateau (TP) during the extended summer season (April-September). Most TPVs stay on the TP, but a small number can move off the TP to the east. TPVs are known to be one of the main precipitation-bearing systems on the TP and moving-off TPVs have been associated with heavy precipitation and flooding downstream of the TP (e.g., in Sichuan province or over the Yangtze River Valley). Identifying and tracking TPVs is difficult because of their comparatively small horizontal extent (400-800 km) and the limited availability of soundings over the TP, which in turn constitutes a challenge for short-term predictions of TPV-related impacts and for the climatological study of TPVs.

In this study, (i) manual tracking (MT) results using radiosonde data from a network over and downstream of the TP are compared with (ii) results obtained by an automated tracking (AT) algorithm applied to ERA-Interim data. Ten MT-TPV cases are selected based on method (i) and matched to and compared with the corresponding AT-TPVs identified with method (ii). Conversely, ten AT-TPVs are selected and compared with the corresponding MT-TPVs. In general, the comparison shows good results in cases where the underlying data are in good agreement, but considerable differences are also seen in some cases and explained in terms of differences in the tracking methods, data availability/coverage and disagreement between sounding and ERA-Interim data. Recommendations are given for future efforts in TPV detection and tracking, including in an operational weather forecasting context.
\end{abstract}

Key words: Tibetan Plateau Vortices, Yearbook of TPVs, TRACK, mesoscale cyclones, manual tracking, automated tracking

Citation: Curio, J., Y. R. Chen, R. Schiemann, A. G. Turner, K. C. Wong, K. Hodges, and Y. Q. Li, 2018: Comparison of a manual and an automated tracking method for Tibetan Plateau Vortices. Adv. Atmos. Sci., 35(8), 965-980, https://doi.org/10.1007/s00376-018-7278-4.

\section{Introduction}

Both mechanical and thermal forcing by the Tibetan Plateau (TP) orography are known to profoundly influence the atmospheric circulation and therefore weather and climate over Asia. One characteristic circulation feature are mesoscale cyclones forming over the TP and its eastern leeward slopes (Tao and Ding, 1981). Specifically, so-called Tibetan Plateau Vortices (TPVs) are shallow cyclonic meso- $\alpha$ scale systems that are identified at the $500 \mathrm{hPa}$ level. TPVs have a horizontal scale of around $500 \mathrm{~km}$ and a vertical extent. of approximately $2-3 \mathrm{~km}$. Observational studies have shown that TPVs that develop and move off the TP to the

\footnotetext{
* Corresponding author: Julia CURIO

Email: j.curio@ reading.ac.uk
}

east can be instrumental in the occurrence of severe weather events downstream of the TP, such as windstorms, heavy rain and hail, and thunderstorms, predominantly in the Sichuan Province and Chongqing region (Tao and Ding, 1981; Wang and Orlanski, 1987; Kuo et al., 1988; Bei et al., 2002; Xiang et al., 2013; Chen et al., 2015). For example, Tao and Ding (1981) earlier revealed that when low-level vortices moved off the TP, they might cause heavy rain and severe storms in summer. Also, many heavy rainfall events, such as those during 14-15 July 1979 in the Sichuan basin (Wang and Orlanski, 1987), 20-22 July 1998 near Hubei (Bei et al., 2002), 2126 July 2010 in Sichuan (Xiang et al., 2013), and 10-13 June 2008 in South China (Chen et al., 2015), have shown that vortices emanating from the TP play an important role. Li et al. (2017) showed that TPVs moving off the TP generate conditions favourable for the generation of mesoscale southwest 
vortices, occurring over the eastern and southeastern flanks of the TP, through the strengthening of the cyclonic vorticity, convergence, and ascending motion. This is important since Yu et al. (2016) found that TPVs and southwest vortices often produce heavy rainfall south of the Yellow River, influencing wide areas of China, in cases where they are active at the same time. Therefore, research into TPVs including their structure, development mechanism, and the prediction of their tracks has been a key concern of Chinese meteorologists for decades (Wu and Chen, 1985; Wang, 1987; Chen and Luo, 2003; Yu and Gao, 2009; Li et al., 2011, 2014a, b; Yu et al., 2014, 2016) and some fruitful results have been achieved. $\mathrm{Wu}$ and Chen (1985) tested the mechanical forcing of different orography schemes on the formation of a mesoscale vortex. Wang (1987) revealed that latent heating over the TP during summer is an essential driving force for the development of TPVs. Chen and Luo (2003) pointed out that the merging of the cyclonic vortex with vorticity lumps in the shearing basic flow of positive vorticity is directly responsible for the maintenance and development of the cyclonic vortex. Li et al. (2011, 2014a, b) studied the occurrence frequency of TPVs and pointed out that the atmospheric heat source has an effect on the development and eastward movement of TPVs. Yu and Gao (2009) and Yu et al. $(2014,2016)$ analyzed observational facts and large-scale conditions leading to the moving off of TPVs. These studies help us to further understand the formation and development of TPVs.

Recent studies have connected the occurrence frequency of TPVs, which exhibits active and suppressed periods, to the variability of the large-scale background circulation. Zhang et al. (2014) found that the 10-30-day intraseasonal oscillation (ISO) modulates the occurrence frequency of TPVs, with 10 times more TPVs occurring in the positive phase of the 10-30-day ISO than in the negative phase, which is a step toward understanding the clustering of TPVs. Other large-scale circulation features, such as the strength and position of the subtropical westerly jet ${ }^{\mathrm{a}}$ (CSHT2018 hereafter) and the MJO (Zhao et al., 2016; Li and Zhao, 2017), have also been found to affect the generation and behaviour of TPVs.

Owing to their comparatively small horizontal scale and shallow structure, as well as observational limitations, it is difficult to identify and track TPVs, especially during the early stages of their lifetime when they are located over the TP. Reliable identification and tracking of TPVs is, in turn, a prerequisite both for the operational prediction of TPVs and their impacts, as well as detailed climatological studies of TPVs, and is therefore an active field of research in China and elsewhere. Currently used tracking methods can broadly be divided into (i) a manual method relying on case-by-case analysis by a human expert and observed sounding data, and (ii) automated methods that track TPVs using spatially complete gridded data from meteorological reanalyses or climate models. The manual method is applied operationally by the China Meteorological Administration (CMA) Chengdu Insti- tute of Plateau Meteorology (IPM) and the results have been published in TPV yearbooks for the years 2000 to 2014 (Li et al., 2000, 2001, 2002, 2003, 2004, 2005, 2006, 2007, 2008; IPM and CMA, 2009, 2010, 2011, 2012, 2013, 2014). The manual tracking method is described in detail in section 2.1.

Several studies have employed automated tracking methods for case studies or to obtain climatologies of TPVs. Recently, Lin (2015) used an objective identification and tracking algorithm to investigate TPV activities based on $500 \mathrm{hPa}$ height data from the ERA-Interim dataset during 1979-2013. The tracks of Lin's method reveal variations in TPV frequency, intensity, persistence, geographical location and migration; but, compared to the paths shown in TPV yearbooks, some significant differences are found, leading Lin (2015) to suggest that improvements of the tracking algorithm are still needed.

Feng et al. (2014) identified TPVs in the $500 \mathrm{hPa}$ streamline and relative vorticity fields of the NCEP-CFSR reanalysis for 10 warm periods (April-October, 2000-09) using a set of three conditions that each detected vortex has to pass to be considered a TPV. They detected $\sim 103$ TPVs on average each year, which mainly occurred between May and August, with the peak count in July. Their results for the geographical genesis distribution of TPVs show that the region of $\left(31^{\circ}-36^{\circ} \mathrm{N}, 84^{\circ}-94^{\circ} \mathrm{E}\right)$ produces two thirds of all TPVs.

A climatology of TPVs based on an ensemble of multidecadal simulations with a $25 \mathrm{~km}$ atmospheric global climate model (GCM) has recently been derived in a companion study (CSHT2018) to this paper, using the TRACK algorithm (Hodges, 1994). TRACK has been used for a wide range of cyclones in the past. Extratropical as well as tropical cyclones have been analyzed in reanalysis datasets (Hoskins and Hodges, 2002; Hodges et al., 2011; Hodges et al., 2017) and climate models (Bengtsson et al., 2007; Catto et al., 2010, 2011, Zappa et al., 2013) looking at the general representation of these systems and changes under future climate conditions. TRACK has also been used to identify and track medicanes ("Mediterranean hurricanes"; Tous et al., 2016) and polar lows (Zappa et al., 2014), which are both mesoscale cyclones like TPVs. The method as applied herein to TPVs is described in detail in section 2.2.

In the companion study (CSHT2018), the GCM is evaluated by applying the same TRACK setup to the same field (filtered $500 \mathrm{hPa}$ relative vorticity) in the model and in two reanalysis datasets. It might be argued, however, that especially over a data sparse region such as the TP, the verifying reanalyses are weakly constrained by assimilated observations and therefore largely depend on the numerical model used in the data assimilation system. It is therefore desirable to also consider an independent TPV climatology that is not derived from reanalysis data, as has also been done for other types of cyclones [e.g., polar lows (Noer et al., 2011), medicanes (Tous and Romero, 2013), tropical cyclones (Bengtsson et al., 2007)]. The IPM TPV yearbooks offer this opportunity,

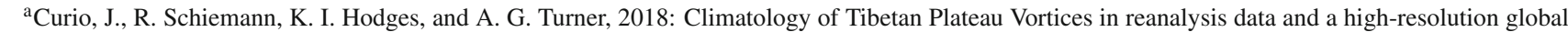
climate model. J. Climate, in revision. 
though they may also suffer from shortcomings, as discussed above.

Accordingly, the aim of this study is to compare TPVs from the IPM yearbooks with those identified by applying TRACK to ERA-Interim data (Dee et al., 2011). We first select 10 examples of TPVs from the yearbooks and match and compare them to the automatically derived reanalysis TPVs. Then, we reverse the approach and select 10 examples of automatically derived TPVs and compare them with the corresponding dates in the TPV yearbooks. We aim to document where the two methods agree and to understand differences in the TPV tracks arising from the chosen methodology or in terms of differences in the underlying data (wind fields from observations and reanalysis data) used by the two methods.

The manuscript is structured as follows: Section 2 gives an overview of the data used, describes the two different tracking approaches in detail, and explains the criteria for the selection of the 20 TPV case studies. The results of the comparison of the manual/subjective and automated/objective tracking approaches are presented in section 3. Differences between the results, possible reasons for the differences and notable issues are discussed in section 4. Section 5 summarizes the results and draws conclusions.

\section{Data and methods}

\subsection{Manual TPV tracking}

The manual subjective TPV tracking uses wind field data from sounding stations as its underlying data. We first ensure that there is an observed cyclonic circulation within the TP region, according to the central position of the cyclone. Next, we track and identify the specific longitude and latitude values for TPVs, the center position being defined generally near the center of the cyclonic wind field, and obtain a sequence of TPV locations for tracking. The specific steps are: (a) When the $500 \mathrm{hPa}$ wind measurement of three or more sounding stations in the TP region together indicate a counterclockwise location, the circulation is defined as belonging to a TPV; (b) The longitude and latitude values of the TPV center drawn between the sounding stations are used to characterize the TPV position, $P_{i}=\left(\operatorname{lat}_{i}, \operatorname{lon}_{i}\right)$, where $i=1,2,3, \ldots, t$ is the time step in question. $P_{i}$ is the TPV location at the $i$ th time; (c) The minimum geopotential height value (GHV) at $500 \mathrm{hPa}$ taken from the sounding stations is used to define the intensity of the TPV circulation $\left(I=G H V_{\text {min }}\right)$. However, a drawback in this method is that it cannot objectively determine the position of the TPV center. In the TPV yearbooks, researchers determine the position of the TPV center mainly according to the above three conditions combined with expert analysis.

\subsection{Automated TPV tracking}

The second method used to identify and track TPVs in this study is an automated objective feature-tracking algorithm, TRACK, developed by Hodges (1994, 1995, 1999). Since it is the first time TRACK has been used to analyze TPVs, the settings used in this study are the same as those in the application of TRACK to polar lows (Zappa et al., 2014), due to their similar spatial scale.

TRACK is applied to the six-hourly $500 \mathrm{hPa}$ relative vorticity field, derived from the ERA-Interim wind field (underlying data). The ERA-Interim data have a horizontal resolution of approximately $0.7^{\circ} \times 0.7^{\circ}(80 \mathrm{~km})$ using the N128 Gaussian grid. Despite general shortcomings of reanalysis data over regions with a low-density station network, such as the TP, ERA-Interim is suitable for this study since it has been used before to analyze TPVs and obtain a TPV climatology (Lin, 2015). ERA-Interim is also known to be able to reproduce the climatology and interannual variability of precipitation in the tropics, e.g., global monsoon precipitation (Lin et al., 2014) and East Asian summer monsoon precipitation (Huang et al., 2016), which might be due to the advanced 12-h 4DVAR data assimilation method (Lin et al. 2014). Chen et al. (2014) found that ERA-Interim, as well as three other reanalyses, reproduce well both the broad pattern and seasonal progress of the rainfall diurnal cycle of East Asia (including the eastern TP) compared with satellite rainfall estimates. Bao and Zhang (2013) compared four reanalyses against independent (not assimilated in the reanalysis) sounding observations. They found that the newer generation reanalyses, ERA-Interim and NCEP-CFSR, have smaller root-mean-square errors than their predecessors.

TPVs are identified and tracked as maxima in the relative vorticity field that has been spatially spectrally filtered. The spectral filtering removes total wavenumbers smaller than 40 (large spatial scale, $>1000 \mathrm{~km}$ ) and larger than 100 (small spatial scale, $<400 \mathrm{~km}$ ), hence allowing TPVs to pass through. The spectral coefficients are also tapered using the method of Sardeshmukh and Hoskins (1984) to further reduce noise in the data, such as Gibbs oscillations. Reducing noise in the very noisy vorticity field results in more reliable identification and tracking. The choice of $500 \mathrm{hPa}$ is important since data presented on pressure levels lower in the troposphere may be intermittently or entirely obscured by orography.

The cyclones are identified as the off-grid maxima greater than $2 \times 10^{-5} \mathrm{~s}^{-1}$ using B-spline interpolation and a steepest ascent method (Hodges, 1995).

The tracking proceeds by first initializing tracks by connecting the maxima using a nearest-neighbor method. These tracks are then refined by minimizing a cost function for track smoothness subject to adaptive constraints, suitably chosen, for the track smoothness and displacement distance in a given time step (Hodges, 1999). At this stage all vorticity maxima are tracked before other criteria are applied to identify the TPVs; this approach maximizes the proportion of the TPV lifecycle that is identified.

The resulting cyclone tracks are then further restricted using three filtering steps: (i) Cyclones that do not originate on the TP, using the $3000 \mathrm{~m}$ altitude line as the reference boundary, are excluded from the data base. This altitude threshold helps to exclude western disturbances (Dimri et al., 2015), which also occur at the $500 \mathrm{hPa}$ level, travelling eastward along the southern slopes of the Himalaya and embedded in 
the Subtropical Westerly Jet; (ii) Only TPVs persisting for at least four time steps (one day) are retained; (iii) Finally a geopotential minimum filter is applied so that an associated geopotential minimum has to occur at least at one time step per TPV within a radius of $5^{\circ}$ around the track point.

\subsection{Selection of TPV cases}

The TPV yearbooks of 2000-14 collected a total of 640 cases, but only a small number of cases were found to move off the TP and trigger heavy rainfall in downstream regions. Figure 1 shows the three main pathways associated with rainfall-namely, east, northeast, and southeast. There are 33 cases moving off the TP along the east path, 22 along the northeast path, and 18 cases moving off along the southeast path. This shows that the TPVs able to move off the TP and cause rainstorms downstream of the TP only account for $13 \%$ of all detected TPVs. It can also be seen that the east path of TPVs mainly affects the region located between the Yangtze and Yellow rivers, the northeast path mainly affects the Yellow River region and parts of northern China, while the southeast path mainly affects Southwest China. The range of TPV activity is large: they can travel as far east as $135^{\circ} \mathrm{E}$, while they occur between $20^{\circ}$ to $45^{\circ} \mathrm{N}$. The domain of interest for the purposes of tracking can therefore be limited to $\left(20^{\circ}-45^{\circ} \mathrm{N}, 80^{\circ}-140^{\circ} \mathrm{E}\right)$. Within this range, 10 representative
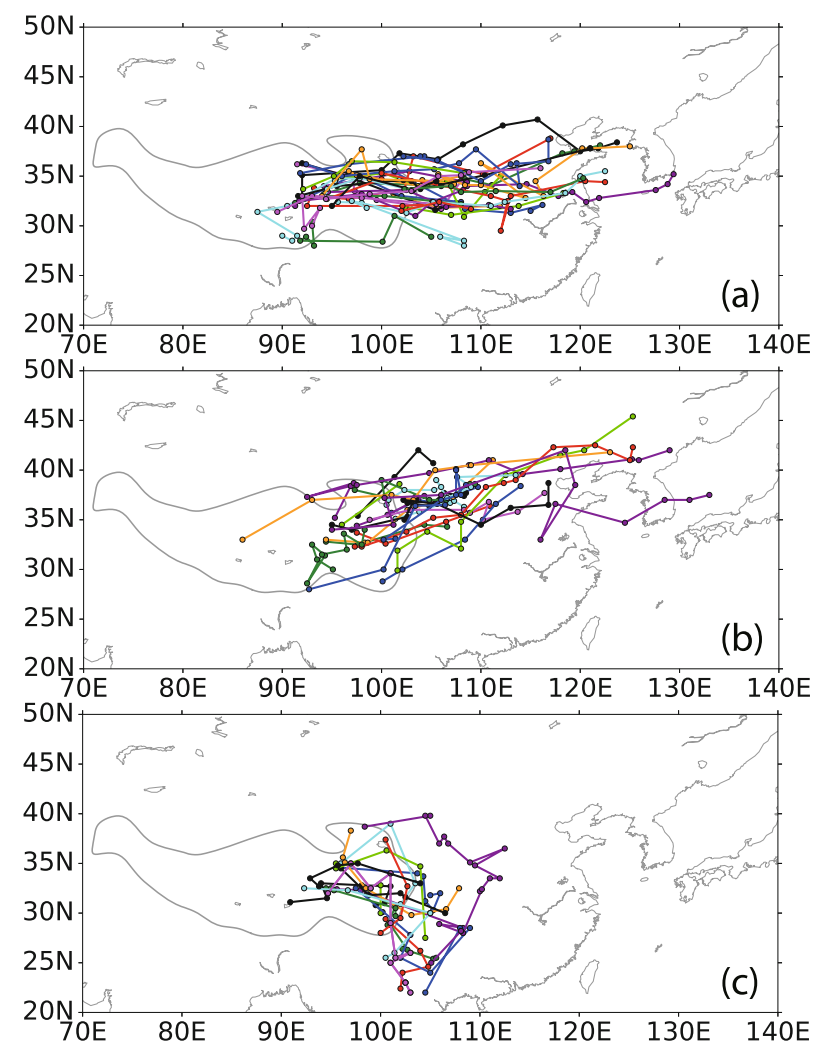

Fig. 1. Main path distribution of TPVs from the IPM yearbooks associated with heavy rainfall for 2000-14: (a) east path (33 cases); (b) northeast path (22 cases); (c) southeast path (18 cases). Each color represents a separate TPV track, with dots at each 12-hourly track point.
TPV cases (Table 1) are chosen from the yearbook of TPVs for a careful comparison between the results of the two different identification and tracking methods. Three selection criteria are applied to choose the 10 cases: (i) the TPVs last for more than two days; (ii) the TPVs move off the TP to the east; and (iii) heavy precipitation $\left(\geqslant 50 \mathrm{~mm} \mathrm{~d}^{-1}\right)$ has to have occurred along the tracked path. In addition, to distinguish between the three different pathways, the longitude of track termination in the east has to exceed $110^{\circ} \mathrm{E}$; the final latitude tracked along the northeast path must exceed $38^{\circ} \mathrm{N}$, and the end latitude of the southeast path must be south of $26^{\circ} \mathrm{N}$.

In fact, the observed tracks from the TPV yearbooks only cover the area east of $90^{\circ} \mathrm{E}$, because there are no viable sounding data available to track TPVs west of $85^{\circ} \mathrm{E}$ (Fig. 2). However, the ERA-Interim data cover the entire plateau area and the main genesis region of TPVs identified in ERAInterim is located in the northwestern $\mathrm{TP}\left(34^{\circ} \mathrm{N}, 84^{\circ} \mathrm{E}\right)(\mathrm{Lin}$, 2015; CSHT2018).

For the complementary comparison of automated tracking results in ERA-Interim to the yearbooks, we also selected 10 cases (Table 1), which (i) moved off the TP to the east, passing $105^{\circ} \mathrm{E}$, and (ii) were associated with heavy precipitation for at least one time step during their lifetime. Heavy precipitation is defined as a precipitation rate above the 95th percentile threshold of all precipitation rates associated with TPV occurrence averaged within $2.5^{\circ}$ around the TPV position.

\section{Results}

\subsection{TPV yearbook manual tracks versus TRACK auto- mated tracks}

In the below analysis we present a visual comparison for the TPV yearbook tracks against those output from the automated feature-tracking algorithm TRACK; comparisons are organized by each of the three path directions-eastward, northeastward and southeastward.

\subsubsection{Eastward path}

Figure 3 shows the tracking result of TPVs with an eastward path. In two cases (case 2, Fig. 3b, and case 4, Fig. 3d)

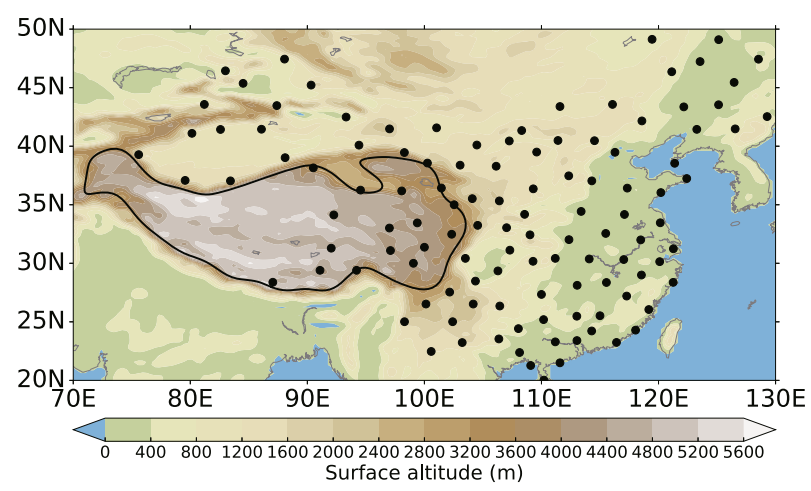

Fig. 2. Distribution of sounding stations (black dots) on the TP and its surroundings used for the manual TPV tracking. The color shading shows the underlying orography (units: $\mathrm{m}$ ). 
Table 1. Two sets of 10 TPV cases, selected from the MT-TPVs, cases 1-10, and from the AT-TPVs, cases 11-20.

\begin{tabular}{|c|c|c|c|c|c|c|}
\hline \multicolumn{7}{|c|}{ MT-TPVs from yearbooks (sounding observations) } \\
\hline No. & ID & Timespan & Year & Path & Longitudinal range & Latitudinal range \\
\hline 1 & $\mathrm{C} 1413$ & 1200 UTC 28 May-1200 UTC 2 June & 2014 & East & $92.0^{\circ}-123.7^{\circ} \mathrm{E}$ & $32.8^{\circ}-38.4^{\circ} \mathrm{N}$ \\
\hline 2 & $\mathrm{C} 1323$ & 1200 UTC 4 June-0000 UTC 10 June & 2013 & East & $94.2^{\circ}-129.4^{\circ} \mathrm{E}$ & $34.0^{\circ}-35.5^{\circ} \mathrm{N}$ \\
\hline 3 & $\mathrm{C} 0726$ & 1200 UTC 6 June-1200 UTC 13 June & 2007 & East & $96.7^{\circ}-125.0^{\circ} \mathrm{E}$ & $32.8^{\circ}-38.0^{\circ} \mathrm{N}$ \\
\hline 4 & $\mathrm{C} 0831$ & 0000 UTC 20 July-0000 UTC 23 July & 2008 & East & $95.4^{\circ}-112.0^{\circ} \mathrm{E}$ & $30.9^{\circ}-35.3^{\circ} \mathrm{N}$ \\
\hline 5 & $\mathrm{C} 1126$ & 1200 UTC 24 June-0000 UTC 27 June & 2011 & Northeast & $94.4^{\circ}-111.2^{\circ} \mathrm{E}$ & $32.7^{\circ}-41.0^{\circ} \mathrm{N}$ \\
\hline 6 & $\mathrm{C} 1026$ & 1200 UTC 17 July-1200 UTC 19 July & 2010 & Northeast & $100.1^{\circ}-114.0^{\circ} \mathrm{E}$ & $28.8^{\circ}-38.4^{\circ} \mathrm{N}$ \\
\hline 7 & $\mathrm{C} 0730$ & 1200 UTC 19 June-1200 UTC 25 June & 2007 & Northeast & $92.5^{\circ}-129.0^{\circ} \mathrm{E}$ & $35.2^{\circ}-42.0^{\circ} \mathrm{N}$ \\
\hline 8 & $\mathrm{C} 1332$ & 0000 UTC 4 July-1200 UTC 7 July & 2013 & Southeast & $98.6^{\circ}-105.6^{\circ} \mathrm{E}$ & $25.4^{\circ}-32.1^{\circ} \mathrm{N}$ \\
\hline 9 & $\mathrm{C} 0739$ & 0000 UTC 30 July-0000 UTC 4 August & 2007 & Southeast & $94.7^{\circ}-103.0^{\circ} \mathrm{E}$ & $22.0^{\circ}-35.0^{\circ} \mathrm{N}$ \\
\hline 10 & $\mathrm{C} 0430$ & 0000 UTC 3 August-0000 UTC 6 August & 2004 & Southeast & $97.5^{\circ}-108.0^{\circ} \mathrm{E}$ & $22.0^{\circ}-32.5^{\circ} \mathrm{N}$ \\
\hline \multicolumn{7}{|c|}{ AT-TPVs from TRACK (ERA-Interim) } \\
\hline No. & ID & Timespan & Year & Path & Longitudinal range & Latitudinal range \\
\hline 11 & 5916 & 0000 UTC 24 June-1800 UTC 26 June & 2008 & East & $84.2^{\circ}-112.2^{\circ} \mathrm{E}$ & $30.5^{\circ}-33.8^{\circ} \mathrm{N}$ \\
\hline 12 & 6310 & 1200 UTC 10 July-1800 UTC 13 July & 2012 & East & $96.7^{\circ}-117.1^{\circ} \mathrm{E}$ & $31.9^{\circ}-34.4^{\circ} \mathrm{N}$ \\
\hline 13 & 6015 & 1200 UTC 29 June-1200 UTC 2 July & 2011 & East & $82.1^{\circ}-108.3^{\circ} \mathrm{E}$ & $33.4^{\circ}-38.7^{\circ} \mathrm{N}$ \\
\hline 14 & 5923 & 1800 UTC 26 June-0000 UTC 30 June & 2003 & East & $83.3^{\circ}-107.4^{\circ} \mathrm{E}$ & $31.8^{\circ}-40.5^{\circ} \mathrm{N}$ \\
\hline 15 & 5097 & 1800 UTC 21 May-0000 UTC 25 May & 2006 & East & $86.8^{\circ}-120.6^{\circ} \mathrm{E}$ & $32.3^{\circ}-36.0^{\circ} \mathrm{N}$ \\
\hline 16 & 5986 & 1800 UTC 27 June-0600 UTC 1 July & 2008 & Northeast & $83.7^{\circ}-118.2^{\circ} \mathrm{E}$ & $33.1^{\circ}-37.3^{\circ} \mathrm{N}$ \\
\hline 17 & 4422 & 1200 UTC 1 May-0000 UTC 6 May & 2010 & Northeast & $89.3^{\circ}-138.5^{\circ} \mathrm{E}$ & $33.0^{\circ}-45.7^{\circ} \mathrm{N}$ \\
\hline 18 & 7328 & 0000 UTC 6 August-1200 UTC 9 August & 2013 & Northeast & $99.2^{\circ}-139.3 .0^{\circ} \mathrm{E}$ & $34.2^{\circ}-43.5^{\circ} \mathrm{N}$ \\
\hline 19 & 5232 & 1200 UTC 11 June-1200 UTC 13 June & 2002 & Southeast & $91.6^{\circ}-105.2^{\circ} \mathrm{E}$ & $29.0^{\circ}-32.6^{\circ} \mathrm{N}$ \\
\hline 20 & 6188 & 1200 UTC 21 June-1200 UTC 24 June & 2010 & Southeast & $92.7^{\circ}-116.3^{\circ} \mathrm{E}$ & $28.7^{\circ}-34.4^{\circ} \mathrm{N}$ \\
\hline
\end{tabular}

the two methods are in good agreement, while there are considerable differences in the other two cases (case 1, Fig. 3a, and case 3, Fig. 3c).

In case 1 (Fig. 3a), the manually tracked (MT) TPV (MT$\mathrm{TPV})$ from the yearbook moves eastward to nearly $125^{\circ} \mathrm{E}$, lasting $132 \mathrm{~h}(11 \times 12$-hourly time steps $)$, while the corresponding automatically tracked (AT) TPV (AT-TPV) from ERA-Interim only lasts $54 \mathrm{~h}(9 \times 6$-hourly time steps $)$. The two tracks also show obvious differences in the TPV path: the yearbook TPV moves northeast while the ERA-Interim TPV moves southeast and is interrupted before moving off the TP. In summary, the tracks do not agree well in case 1.

In case 2 (Fig. 3b) the MT-TPV from the yearbook lasts $144 \mathrm{~h}(12 \times 12$-hourly time steps $)$ while the AT-TPV in ERAInterim lasts $60 \mathrm{~h}(10 \times 6$-hourly time steps). The paths of the TPVs show good agreement, especially west of $120^{\circ} \mathrm{E}$, but the AT-TPV is interrupted at 1800 UTC 6 June, while the MT-TPV still lasts until 0000 UTC 10 June. The start position of the MT-TPV is near $95^{\circ} \mathrm{E}$, while the start position of AT-TPV is slightly further east (near $98^{\circ} \mathrm{E}$ ), and the positions at the same time steps are similar.

In case 3 (Fig. 3c), the MT-TPV and AT-TPV have the same start time, but the location of the TPV at each time step is different. The MT-TPV starts at 1200 UTC 6 June near $95^{\circ} \mathrm{E}$, while the matching AT-TPV starts 24 hours ear- lier at $85^{\circ} \mathrm{E}$. While the TPV moves off the TP at $0000 \mathrm{UTC}$ $18 \mathrm{July}$, the MT-TPV lasts $131 \mathrm{~h}(11 \times 12$-hourly time steps $)$ and reaches $125^{\circ} \mathrm{E}$, but the AT-TPV is interrupted after 0000 UTC 18 July.

In case 4 (Fig. 3d), the AT-TPV starts at 1200 UTC 18 July near $80^{\circ} \mathrm{E}$ and the MT-TPV starts at 0000 UTC 20 July. Once the TPV moves east of $90^{\circ} \mathrm{E}$, it is also seen in the MT-TPV and the location of the TPV at corresponding time steps shows only small differences between the two methods. However, the AT-TPV lasts longer than the MT-TPV and travels a larger distance, reaching the Bohai Sea in the northern part of the Yellow Sea.

\subsubsection{Northeast path}

Three cases are selected from the yearbook to represent the northeast path, two of which (case 5 and case 7) have matching results in ERA-Interim (Fig. 4). Figure 4a shows the MT-TPV from the yearbook and the AT-TPV from ERAInterim for case 5, which was first detected at 1200 UTC 24 June in the yearbook, and 24 hours earlier (1200 UTC 23 June) and further west (to the west of $90^{\circ} \mathrm{E}$ ) in ERA-Interim. During their overlap time, both tracks correspond well and at some time steps the positions of the identified TPV are basically the same. However, obvious differences between the two tracks are found after 1200 UTC 26 June-namely, that 


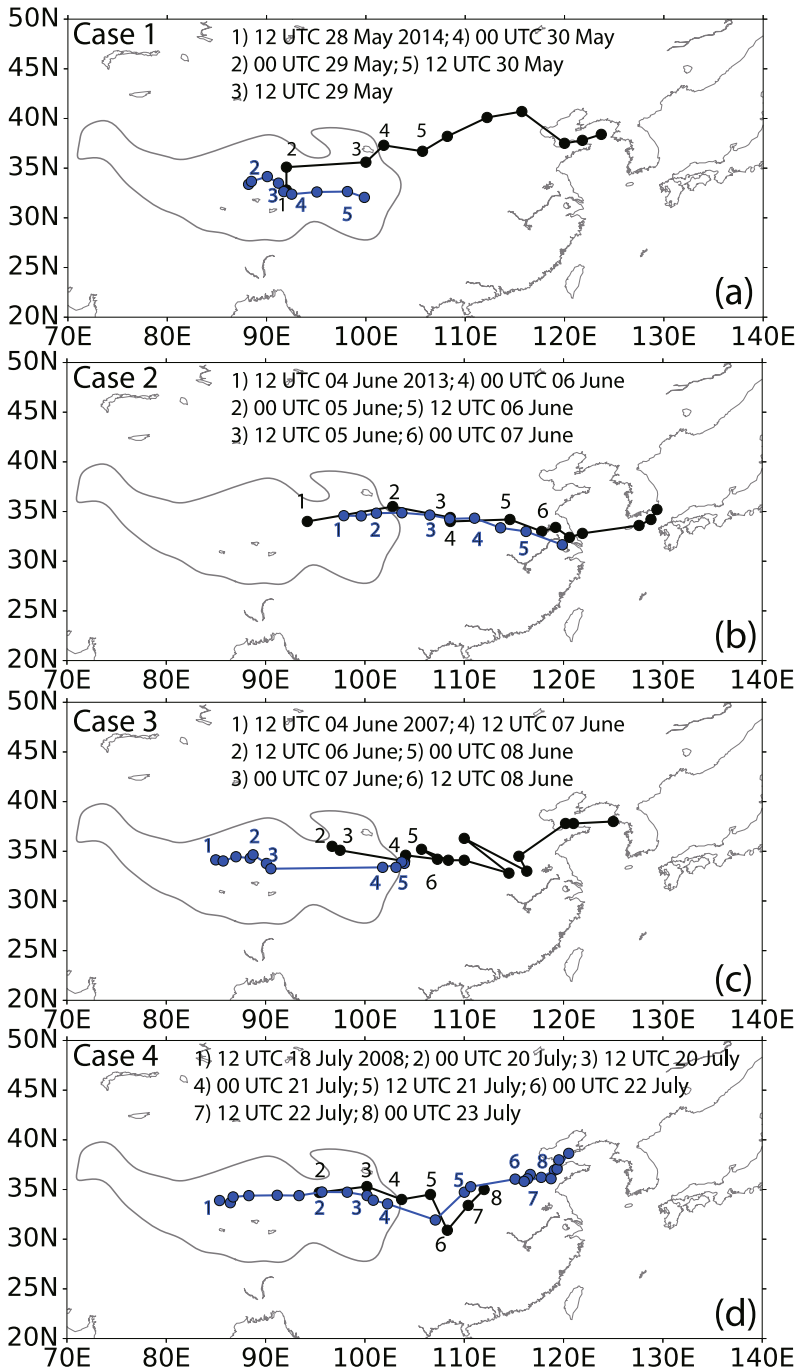

Fig. 3. Tracks for the four TPV cases with an east path selected from the TPV yearbooks (black dots) and the matching TPVs output from the AT algorithm TRACK (blue dots): (a) case 1; (b) case 2; (c) case 3; (d) case 4 .

the AT-TPV moves toward the southeast while the MT-TPV continues to move northeastward.

For case 7 (Fig. 4b), both tracks show the characteristics of the northeast path. Over the TP the AT-TPV is located further south compared to the MT-TPV, but the two tracks tend to coincide after moving off the TP. The AT-TPV is interrupted after 1200 UTC 24 June, while the MT-TPV lasts until 1200 UTC 25 June. Overall, the paths overlap/agree east of the TP until the AT-TPV is interrupted, but the start and end dates and locations exhibit large differences.

\subsubsection{Southeast path}

Figure 5 shows the tracks of the MT-TPV and AT-TPV for TPV cases with southeast paths. For the southeast-path TPVs, the comparison between MT-TPVs and AT-TPVs shows overall larger differences than for the east and northeast paths.

In case 8 (Fig. 5a) both methods detect TPVs but the paths and identified positions are different and the AT-TPV track is interrupted when the TPV moves off the TP to the south at a

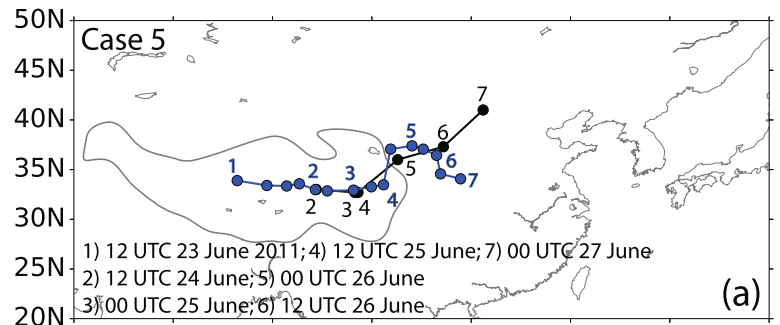

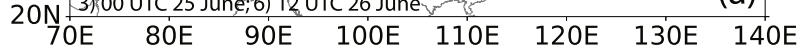

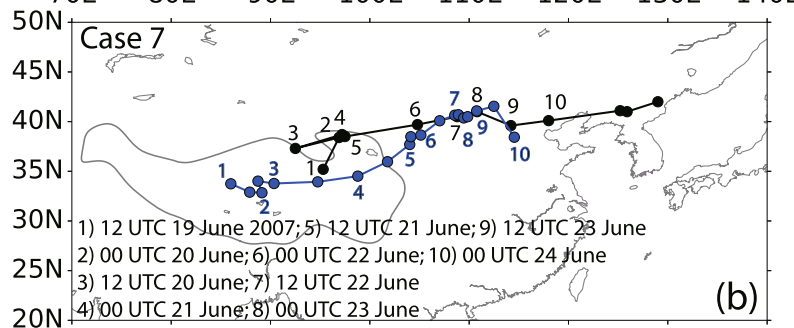

$20 \mathrm{~N}$ 4) (00 UTC 21 June; 8) 0Q. UTC 23 June

Fig. 4. Tracks for the two TPV cases with a southeast path selected from the TPV yearbooks (black dots) and the matching TPVs output from the AT algorithm TRACK (blue dots): (a) case 5; (b) case 7 .
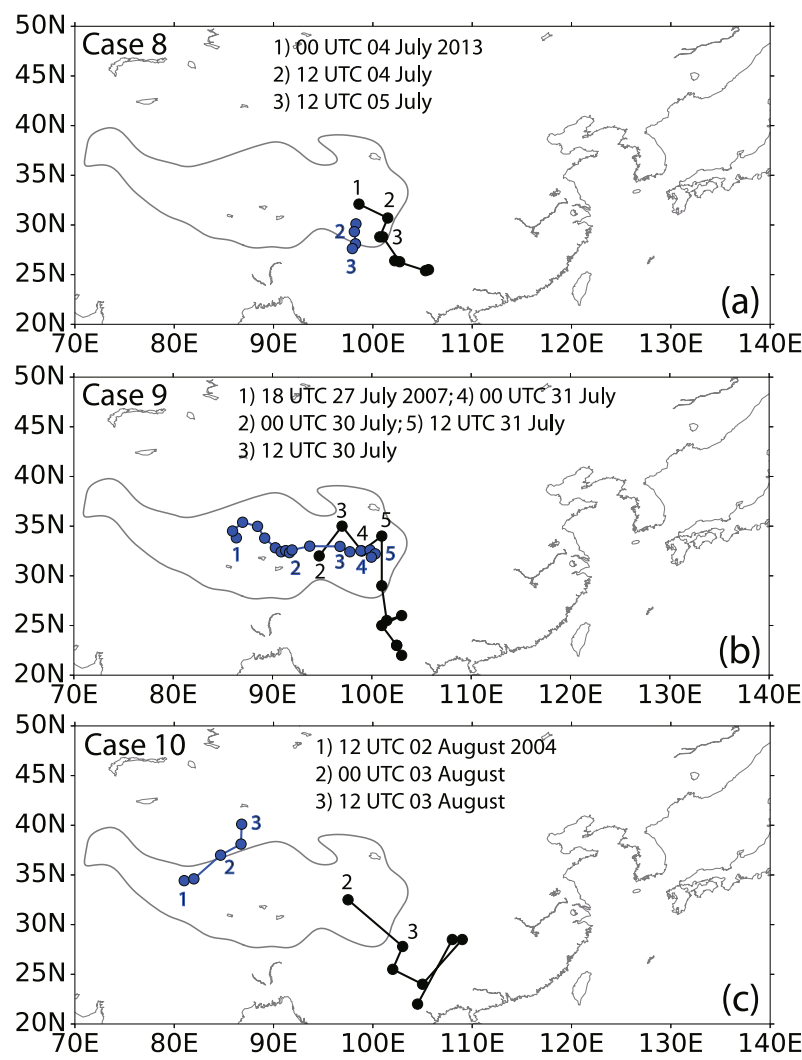

Fig. 5. Tracks for the three TPV cases with a northeast path selected from the TPV yearbooks (black dots) and the matching TPVs output from the AT algorithm TRACK (blue dots): (a) case 8 ; (b) case 9; (c) case 10.

different location than the MT-TPV moving off to the southeast. For the MT-TPV in case 9 (Fig. 5b) a corresponding AT-TPV track is identified starting 30 hours earlier at 0000 
UTC 27 July 2007 and $10^{\circ}$ further west (near $85^{\circ} \mathrm{E}$ ) than the MT-TPV. East of $90^{\circ} \mathrm{E}$, both the AT-TPV and MT-TPV basically have consistent paths for 36 hours $(4 \times 12$-hourly time steps), but the AT-TPV is interrupted when the MT-TPV moves southward after 1200 UTC 31 July.

For case 10 (Fig. 5c), no corresponding TPV is identified in AT and only a TPV at the other end of the TP is found to be occurring simultaneously. This case is rated as a miss.

\subsection{TRACK automated tracks versus TPV yearbook man- ual tracks}

In the below analysis we present a visual comparison for the TPV tracks output from the automated feature tracking algorithm TRACK against those from the TPV yearbooks; comparisons are organized by each of the three path directions - eastward, northeastward and southeastward.

\subsubsection{East path}

After comparing the results chosen from the yearbooks to TRACK, we further compare the results of 10 selected TPV cases using the TRACK AT method in ERA-Interim to the MT events on the same dates from the yearbooks. Figure 6 shows five AT-TPV cases from ERA-Interim with east paths matched to MT-TPVs from the yearbooks.

In case 11 (Fig. 6a), the two tracks show a good matching result and have a consistent path. The AT-TPV lasts $72 \mathrm{~h}(12 \times 6$-hourly time steps), starting in the western TP $\left(\sim 33.2^{\circ} \mathrm{N}, 84^{\circ} \mathrm{E}\right)$ and ending over the mainland of China $\left(\sim 31.7^{\circ} \mathrm{N}, 112^{\circ} \mathrm{E}\right)$. The corresponding MT yearbook TPV starts later and further east $\left(33.4^{\circ} \mathrm{N}, 94.6^{\circ} \mathrm{E}\right)$ than the AT-TPV, but ends at a very similar position $\left(31.7^{\circ} \mathrm{N}, 113^{\circ} \mathrm{E}\right)$, lasting $60 \mathrm{~h}(5 \times 12$-hourly time steps). In case 12 (Fig. 6b), the ATTPV has a long track, but the MT-TPV is interrupted before moving off the TP. Over the TP the two paths are matching, but the MT-TPV only lasts $48 \mathrm{~h}(4 \times 12$-hourly time steps $)$ and does not move off the TP, while the AT-TPV lasts $84 \mathrm{~h}$ (14×6-hourly time steps), and travels off the TP and close to the coast $\left(33.75^{\circ} \mathrm{N}, 117.14^{\circ} \mathrm{E}\right)$. In case 13 (Fig. $6 \mathrm{c}$ ), the ATTPV is again identified earlier than the corresponding MTTPV (compare to case 1). The AT-TPV starts two days prior to the MT-TPV, but for the overlapping time steps both paths are similar. In case 14 (Fig. 6d) the paths of the AT-TPV and the MT-TPV show large differences, especially downstream of the TP. For example, after 0000 UTC 29 June 2003, the AT-TPV moves back to the TP, but the MT-TPV continues moving eastward. In case 15 (Fig. 6e), the AT-TPV lasts $84 \mathrm{~h}$ ( $14 \times 6$-hourly time steps), while the MT-TPV is only identified at two 12-hourly time steps within this period.

In summary, the AT-TPVs generally have longer tracks and the region of activity is located further west than for the MT-TPV. However, in some cases where both TPVs occur simultaneously, the tracking results are similar.

\subsubsection{Northeast path}

Three cases of northeast TPV paths from TRACK (ATTPVs) are chosen to match MT- TPVs (Fig. 7). Just as for

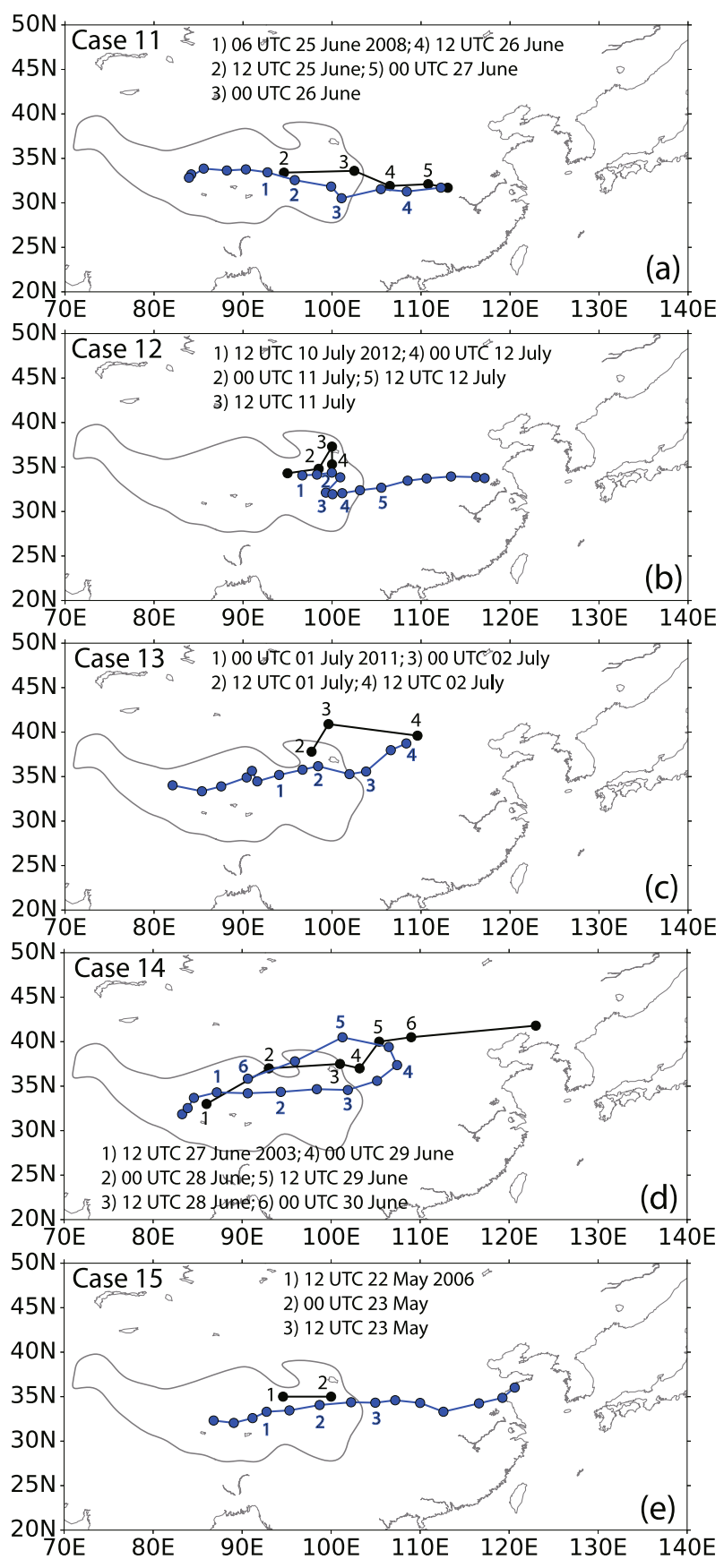

Fig. 6. Tracks for the five TPV cases with an east path selected from the results of automated TPV tracking with the TRACK algorithm (blue dots) and the matching TPVs from the TPV yearbooks (black dots): (a) case 11; (b) case 12; (c) case 13; (d) case 14; (e) case 15.

the east path, the AT-TPVs have longer paths than the MTTPVs. For example, the AT-TPV in case 16 (Fig. 7a) lasts 90 h $(15 \times 6$-hourly time steps $)$ but is only detected at two time steps (24 h) using MT. During the overlap the TPV paths are consistent. In case 17 , the AT-TPV shows a very long track traveling further east than $135^{\circ} \mathrm{E}$ and lasting for $114 \mathrm{~h}$ ( $19 \times 6$-hourly time steps), but there is no matching MT-TPV in the yearbook. In case 18, there is an MT-TPV identified at 

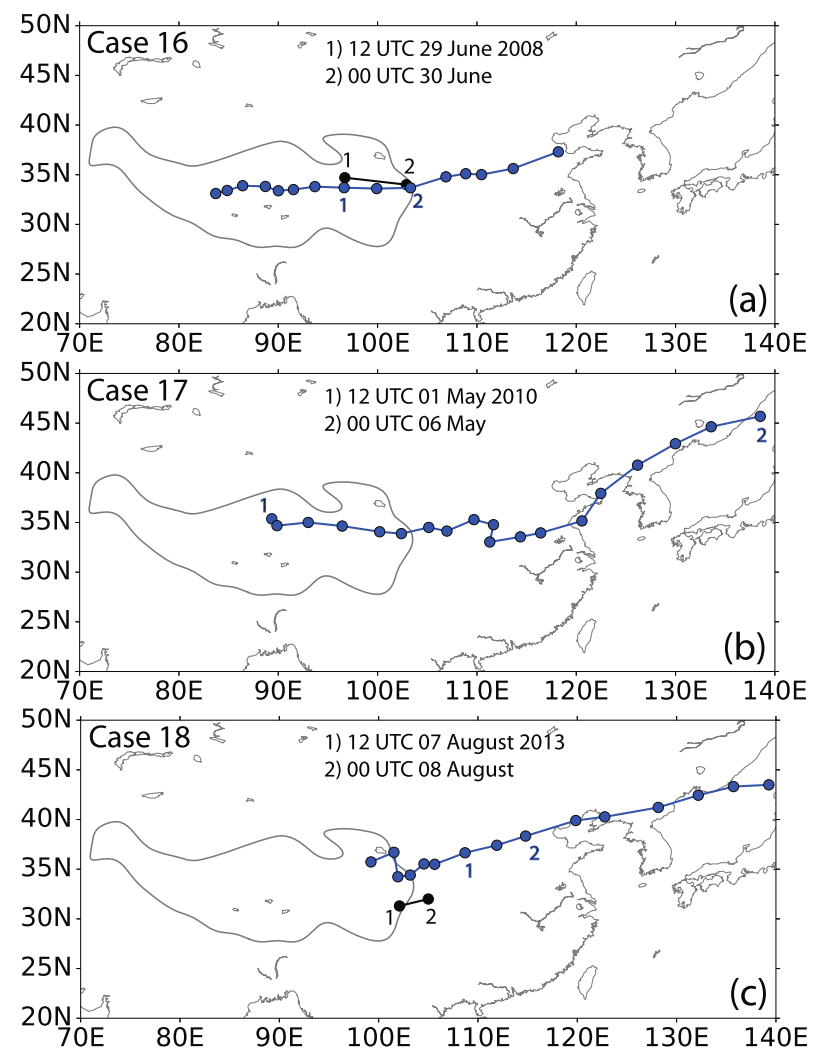

Fig. 7. Tracks for the three TPV cases with a northeast path selected from the results of automated TPV tracking with the TRACK algorithm (blue dots) and the matching TPVs from the TPV yearbooks (black dots): (a) case 16; (b) case 17; (c) case 18.

two time steps but the position is not matching the AT-TPV. Overall, the results of matching northeast-moving AT-TPVs to MT-TPVs from the yearbooks show larger differences than for TPVs moving eastward.

\subsubsection{Southeast path}

Two cases of southeast TPV paths from the AT-TPVs are chosen to be compared to the MT-TPVs from the yearbooks (Fig. 8). In case 19 (Fig. 8a), the matched result is good and the AT-TPV and MT-TPV have similar tracked positions for specific time steps. In case 20 (Fig. 8b), the AT-TPV moves southeast but the corresponding MT-TPV moves eastward. However, when the TPVs are located within/over the TP, the tracked results are basically consistent and acceptable.

\section{Discussion}

The examples shown in section 3 include cases of good agreement between the MT-TPVs and AT-TPVs, but also cases of considerable disagreement. In this section, we discuss some of the reasons for disagreement using three selected cases (cases 5, 1, and 9). We organize the discussion into differences in data availability and resolution (section 4.1), differences between the tracking methods themselves (section 4.2) and differences between the underlying data

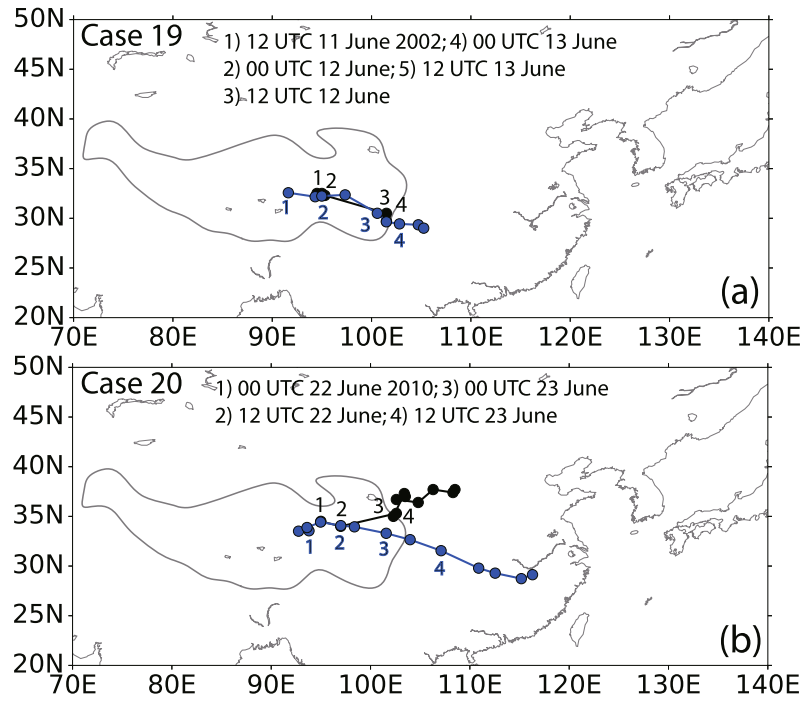

Fig. 8. Tracks for the two TPV cases with a southeast path selected from the results of automated TPV tracking with the TRACK algorithm (blue dots) and the matching TPVs from the TPV yearbooks (black dots): (a) case 19; (b) case 20.

(section 4.3); however, we recognize that several or all of these issues may contribute to the disagreement between MT and AT tracks in individual cases. We also repeat the tracking of TPVs for the three cases using the MT on ERA-Interim data (section 4.4).

\subsection{Differences in data availability and resolution}

It is undeniable that differences exist between the observations and the ERA-Interim data. The observations from the sounding station network are available every 12 hours, and therefore the movement of the TPVs can only be tracked in 12-hourly time steps, while the AT method uses 6-hourly ERA-Interim data to track the TPVs. Therefore, the distance the TPVs can move between two time steps differs between the two methods. This could lead to a misidentification of a system as the same TPV as in the earlier time step using MT.

Because there are no viable sounding stations available in the western part of the TP, the tracking of TPVs using direct observations is limited to the central and eastern part of the $\mathrm{TP}$, and thus only cases occurring in this region are collected in the yearbooks. When we compare the tracking results from the two types of data, some cases show that the AT-TPVs occur earlier than the corresponding MT-TPVs, and the location where the TPVs are identified for the first time is further west on the TP than for the MT method. For example, in cases 4 (Fig. 3d), 5 (Fig. 4a) and 9 (Fig. 5b), the tracks of AT-TPVs start earlier and the start positions are located west of $90^{\circ} \mathrm{E}$ where no observations from sounding stations are available, but MT picks up the TPVs only eastward of $90^{\circ} \mathrm{E}$.

To illustrate the problem, we use case 5 from the yearbooks as an example. Figure 9 shows the TPV's position from AT $(\mathrm{a}-\mathrm{d}, \mathrm{f})$ for $5 \times 6$-hourly time steps and the position from MT for one corresponding time step (e). The AT-TPV starts west of $90^{\circ} \mathrm{E}$ at 1200 UTC 23 June (a) and moves continu- 
(a) 12 UTC 23 June 2011

(b) 18 UTC 23 June 2011

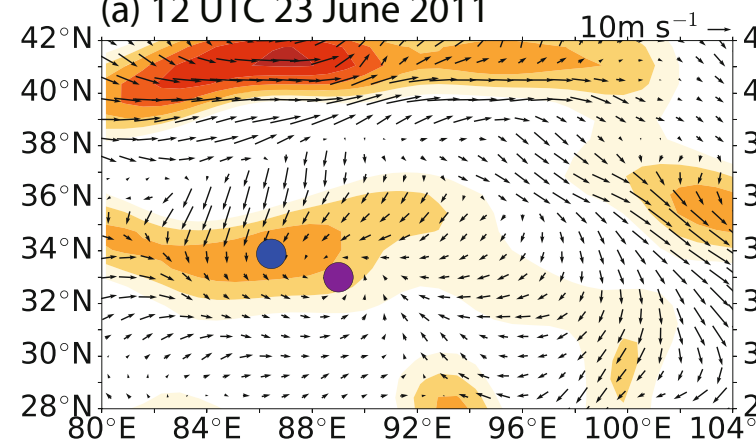

(b) 18 UTC 23 June $2011,10 \mathrm{~m} \mathrm{~s}^{-1} \rightarrow$

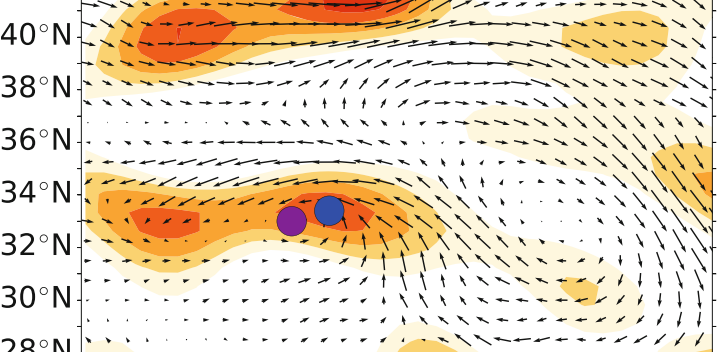

(c) 00 UTC 24 June 2011

$10 \mathrm{~m} \mathrm{~s}-1.72^{\circ} \mathrm{N}$

(d) 06 UTC 24 June 2011

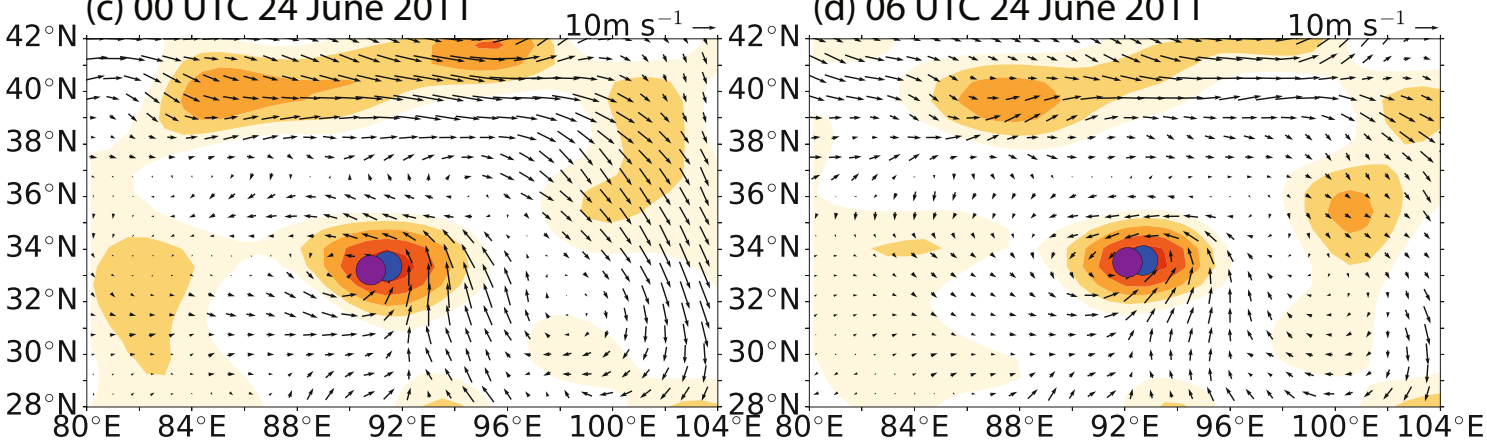

(e) 00 UTC 24 June 2011

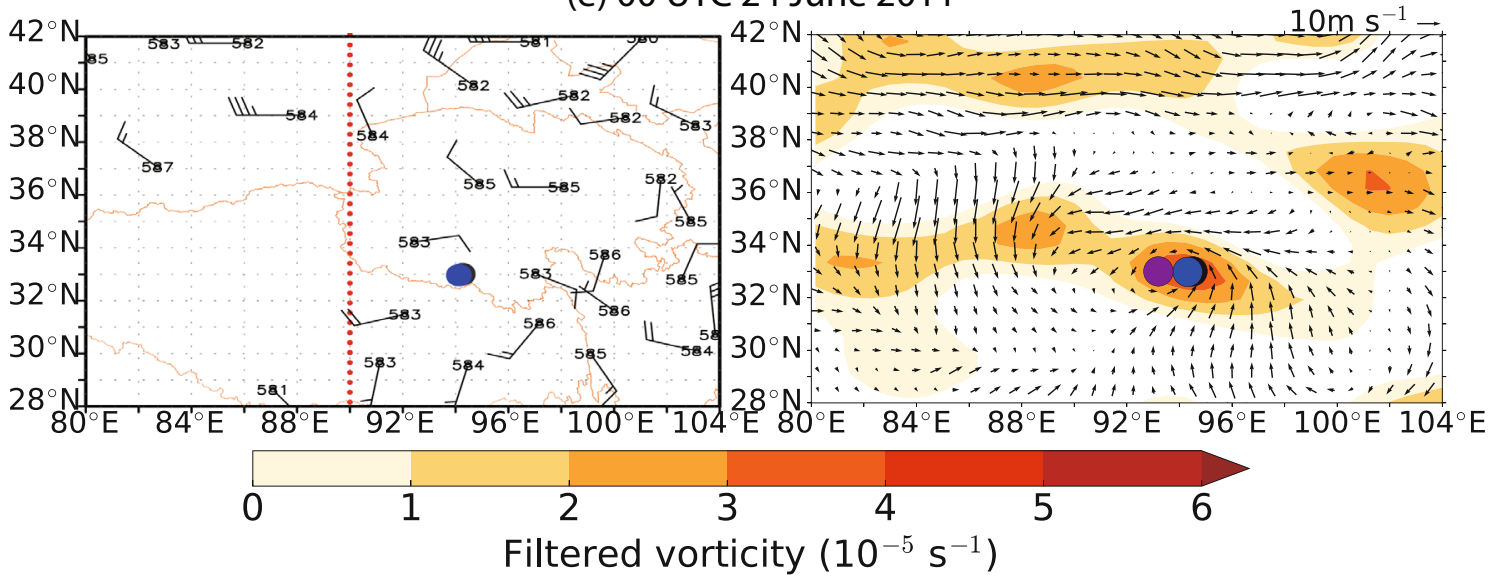

Fig. 9. $500 \mathrm{hPa}$ wind field (vectors) and spectrally filtered relative vorticity (shading) from ERA-Interim [a-d, e (right)] and $500 \mathrm{hPa}$ wind (barbs) and geopotential height (numbers) from the observational data at the sounding stations for selected time steps of case 5. The black dots denote the position of the MT-TPV from the TPV yearbook, while the blue dots denote the position of the AT-TPV using TRACK. The purple dots denote the position of the MT-TPV using ERA-Interim.

ously eastward during the next time steps $(b-d, f)$. The MT method first identifies the TPV at 1200 UTC 24 June (Fig. 9e), once the TPV has moved into the area where sounding stations exist. Both methods agree on the position of the TPV at this time step (e) and also result in similar positions over the further course of the TPV (Fig. 4a). This case demonstrates how data availability can impact TPV identification and tracking.

\subsection{Difference in tracking methods}

The methods used to identify and track TPVs also exhibit differences that can impact the results and lead to differences between the obtained TPV tracks. The first step when manu- ally tracking TPVs is to make sure that there is a cyclonic circulation observed over the TP. The position (longitude, latitude) of the TPV at each 12-hourly time step is then manually determined by an expert according to the respective location of the center of the cyclonic wind field, thereby obtaining a sequence of TPV positions that together form a TPV track. The AT method uses an objective feature-tracking algorithm, TRACK, to identify and track TPVs as maxima in the 500hPa vorticity field from ERA-Interim (6-hourly, spectrally filtered).

Figure 10 shows the difference of the two tracking methods in case 1. At 1200 UTC 28 May 2014 (Fig. 7a), the 500 $\mathrm{hPa}$ wind field from the sounding data shows an incomplete 
cyclone in the westernmost region of the station network and the ERA-Interim wind field shows a weak cyclonic circulation in the same region. At this time step, a track is identified by MT but not by AT. The TPV is identified by AT six hours later at 1800 UTC 28 May around $4^{\circ}$ further west (Fig. 10b) where a strong relative vorticity maximum is visible. In the

(a) 12 UTC 28 May 2014

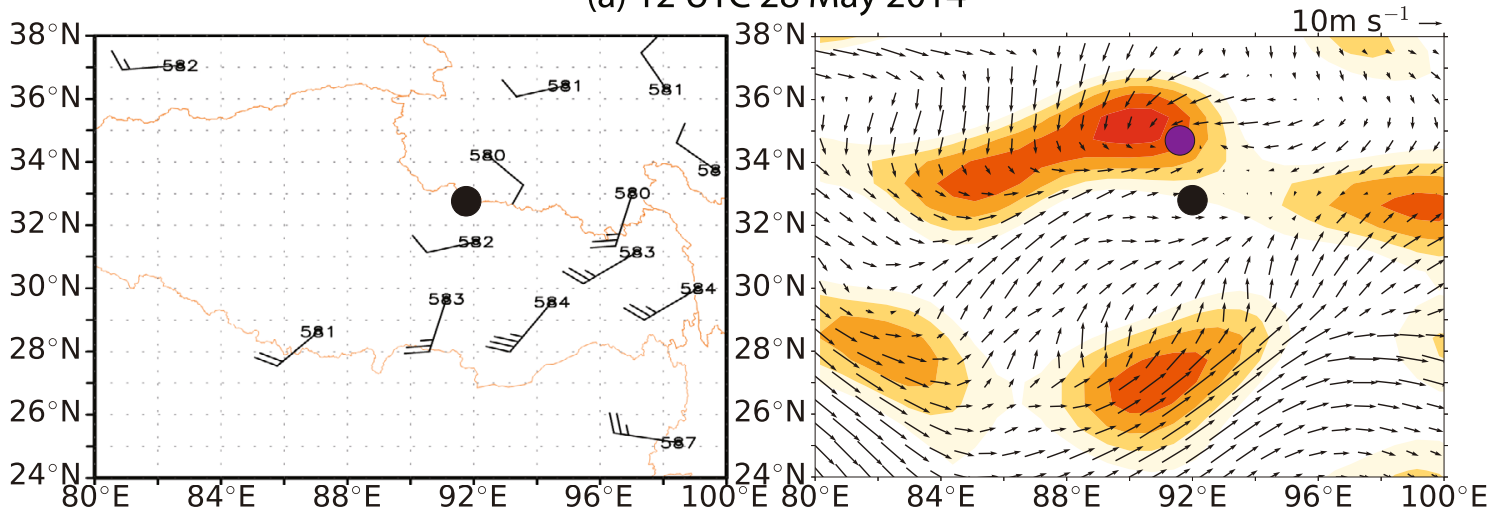

(b) 18 UTC 28 May 2014

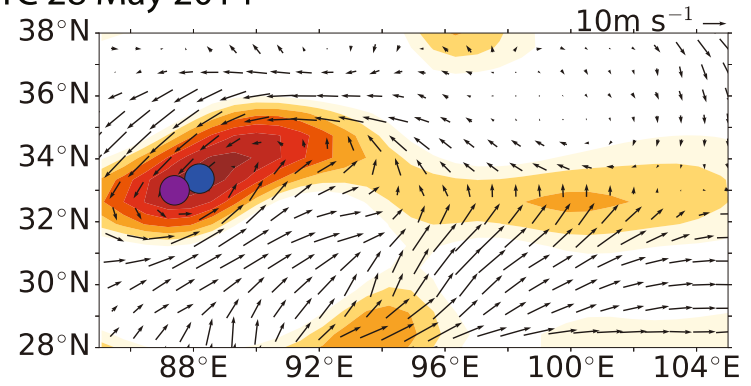

(c) 00 UTC 29 May 2014

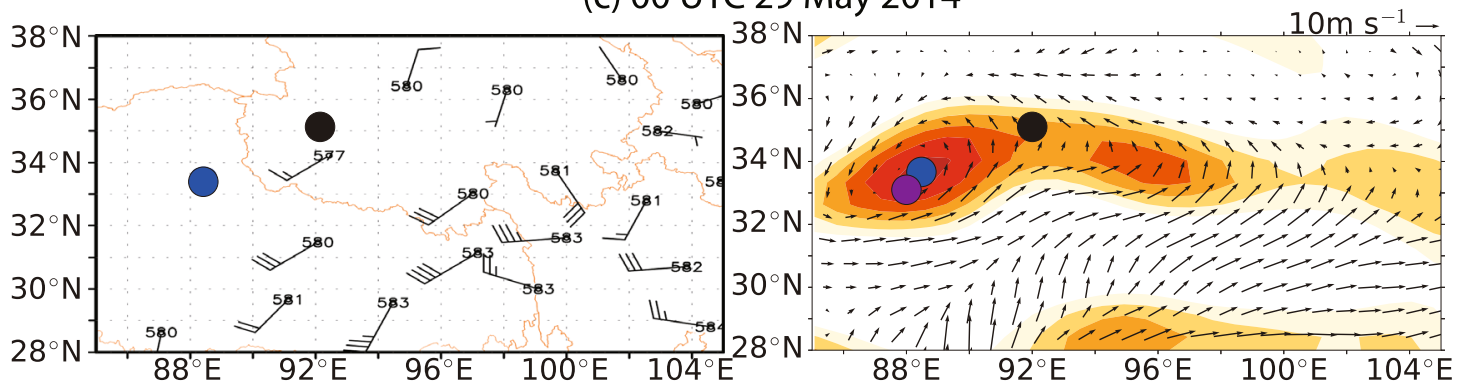

(d) 12 UTC 29 May 2014
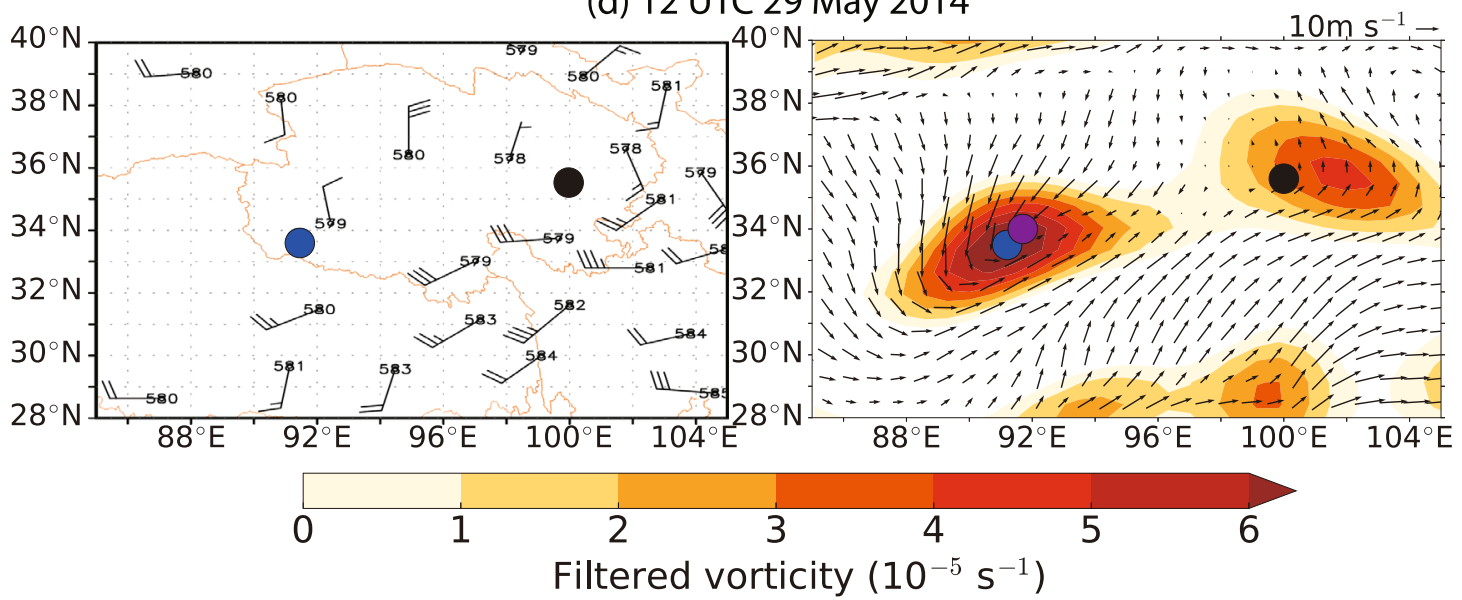

Fig. 10. $500 \mathrm{hPa}$ wind field (vectors) and spectrally filtered relative vorticity (shading) from ERA-Interim (right) and $500 \mathrm{hPa}$ wind (barbs) and geopotential height (numbers) from the observational data at the sounding stations (left) for selected time steps $(\mathrm{a}-\mathrm{g})$ of case 1 . The black dots denote the position of the MT-TPV from the TPV yearbook, while the blue dots denote the position of the AT-TPV using TRACK. The purple dots denote the position of the MT-TPV using ERA-Interim. 
(e) 00 UTC 30 May 2014

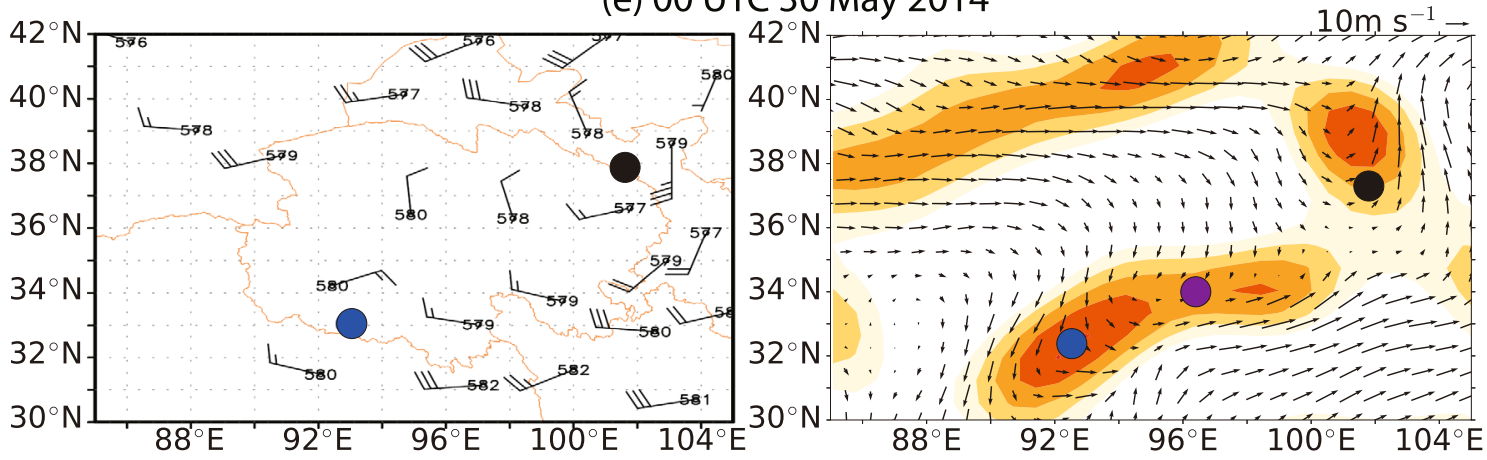

(f) 12 UTC 30 May 2014

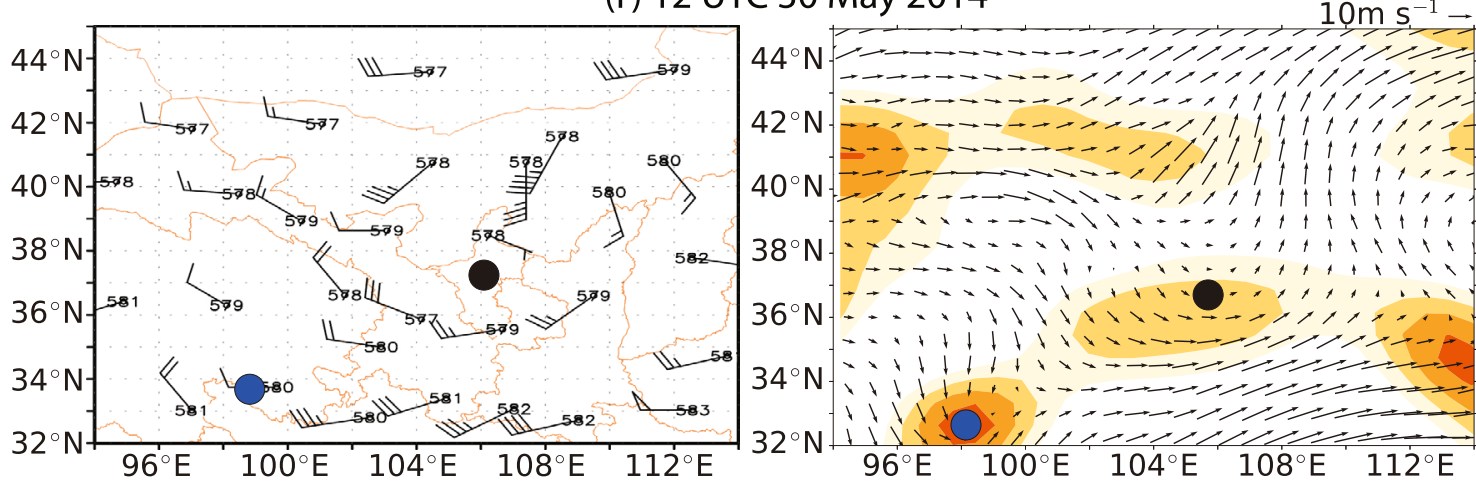

(g) 00 UTC 31 May 2014

$10 \mathrm{~m} \mathrm{~s}^{-1} \rightarrow$

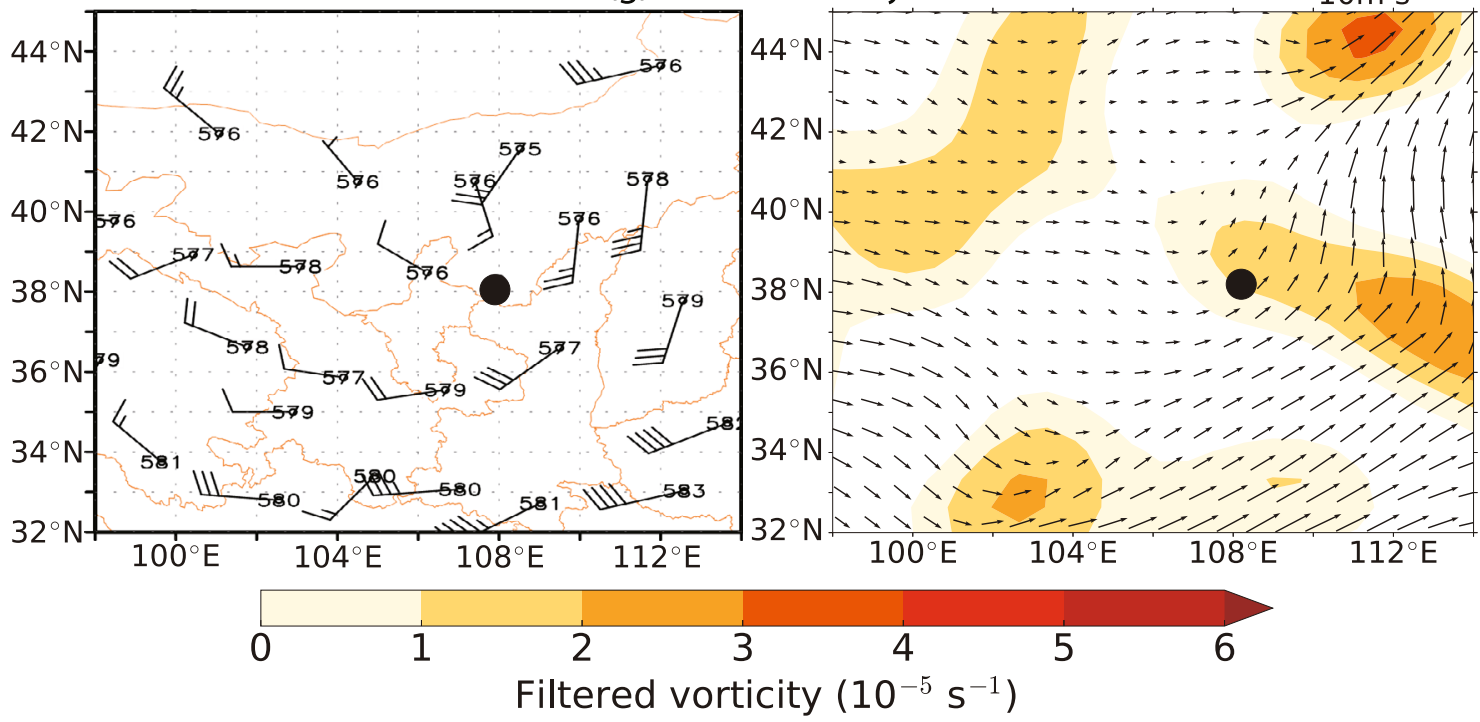

Fig. 10. (Continue.)

later evolution, it becomes clear that the two methods track two different systems. Figure $7 \mathrm{c}$ shows the two identified TPVs relatively close to each other at 0000 UTC 29 May. At this time, the filtered vorticity field shows an area of relatively high vorticity with two local maxima. The AT-TPV is identified at the center of the stronger maximum, while the MT-TPV is located between the two maxima. Twelve hours later, these two local maxima intensify and evolve into two different systems. From then on, the TPV position identified by MT agrees with the position of the second vorticity maximum, which travels eastwards along $36^{\circ} \mathrm{N}$, while the TPV identified by AT travels southeastward. Hence, the differences between the two TPV tracks increase over time (Fig. 3a). The same splitting happens in case 20 (Fig. 8b), where the two methods also track two different systems after they first show very similar positions.

Possible reasons for the second vorticity maximum not being identified as an AT-TPV are differences in the wind fields and/or a track that is too short (an AT-TPV has to persist for at least four time steps). It is also possible that a TPV is identified by AT initially but that the track breaks due to a vorticity anomaly below the threshold used in TRACK. A 
further intensification of the same system will not be identified by TRACK if happening outside the TP area defined by the $3000 \mathrm{~m}$ altitude line.

This could also be the reason for the fact that, in case 10 (Fig. 5), the AT method does not identify a TPV in the eastern part of the TP (Fig. 5c). In such cases, the long-term experience of an expert, familiar with the behavior of TPVs, e.g., incomplete cyclonic circulations, TPV splitting, and expansion, may be an advantage.

These cases also reveal that the settings used in TRACK could be improved, e.g., by adjusting the thresholds used. Another option would be to not discard all cyclones that are identified for the first time only when downstream of the TP, and multiple tracks could also be combined into one. A question arising from this study is also whether TPVs move off the TP and the same system then travels eastward, or if the moving-off TPV triggers the formation of a new cyclonic system. A recent work in this area (Li et al., 2017) suggests that TPVs moving off the TP are favorable for the genesis of southwest vortices, which import water vapor from the south and lead to heavy rainfall.

\subsection{Differences between underlying data}

The two tracking methods produce very similar results in cases where the differences between the observations and ERA-Interim data are small; on the other hand, large differences would lead to different tracking results.

Figure 11 shows the $500 \mathrm{hPa}$ wind field from the observations and ERA-Interim for case 9. The TPVs identified by MT and AT are located in the same region at very similar positions, since the wind field in the area of TPV occurrence is consistent between the two datasets until 1200 UTC 31 July. But, at 0000 UTC 1 August (Fig. 11e), the $500 \mathrm{hPa}$ wind field from ERA-Interim shows only a weak cyclonic circulation and the vorticity maximum has weakened substantially. Therefore, no AT-TPV is identified at this time step and the TPV track breaks, while the MT-TPV starts to travel southward and lasts for another three days.

\subsection{Manual tracking using ERA-Interim}

We repeated the tracking of TPVs in ERA-Interim for three selected cases using the MT approach. The results in Figs. 9, 10 and 11 show that the results from the MT and AT of TPVs in ERA-Interim are consistent when the wind field exhibits an obvious cyclonic circulation (Figs. 9b-e; Figs. 10b-d; Figs. 11a-d). In situations where the cyclonic circulation is less obvious, the identified TPV position shows slightly larger differences between the two methods (Figs. 10a and c), but both methods still identify the same system. Only in situations where the wind field does not exhibit a closed cyclonic circulation, e.g., Fig. 10e, can the TPV position not be identified manually. Whether there is a closed cyclonic circulation visible is highly dependent on the resolution of the dataset. These results give us confidence that the objective feature-tracking algorithm TRACK is suitable for identifying and tracking TPVs in ERA-Interim. In future work the automated tracking could be applied to a reanalysis dataset with a higher horizontal resolution, such as ERA5.

\section{Summary and conclusion}

In this study we use two sets of 10 TPV cases selected from (i) MT-TPVs collected in the IPM yearbooks of TPVs and (ii) AT-TPVs from ERA-Interim using an objective feature-tracking algorithm, TRACK, for an in-depth comparison of tracking results. First, the cases selected from the yearbooks of TPVs are matched to TPVs identified by AT and their tracks are compared. In a second step, we compare the TPVs the other way round, selecting cases from the database of AT-TPVs in ERA-Interim and matching these with cases from the TPV yearbooks.

The 20 TPV cases used in this study can be qualitatively differentiated into three categories (match, partial match, and no match) regarding the level of agreement between the two tracking results. Cases in which the two TPV tracks only agree during part of their lifetime (e.g., they exhibit very similar tracks first but diverge later) are classified as partial matches. Table 2 shows all 20 cases and their assigned matching categories. For the comparison of selected MT-TPVs with AT-TPVs (cases 1-10), the table shows three matches (green), three partial matches (yellow), and four non-matches (red). For the second set of TPVs, AT-TPVs matched to MTTPVs (cases 11-20), the comparison results in two matches (green), six partial matches (yellow), and two non-matches (red).

Differences between the results are related to three types of issues revealed in this study: (i) differences in the data availability and their spatiotemporal resolution; (ii) differences in the tracking methods used; and (iii) differences between the underlying data.

Differences in the data resolution and availability lead to an earlier and further westward detection of TPVs using AT compared to MT, due to the complete spatial coverage in ERA-Interim and the higher temporal resolution. The absence of sounding stations in the western part of the TP and the lower temporal resolution of the observations may cause an under-sampling of TPVs in MT. Additionally, the relatively sparse station network makes it difficult to identify the center of the cyclonic circulation precisely.

Differences related to the tracking methods can lead to the tracking of two separate systems in the latter stages in cases where a TPV splits into two. TRACK will only identify a

Table 2. Categorization of TPV cases selected from MT-TPVs to match with AT-TPVs (cases 1-10) and from the AT-TPVs to match with MT-TPVs (cases 11-20) into three categories: match (green); partial match (yellow); and non-match (red).

\begin{tabular}{ccccccccccc}
\hline \multicolumn{1}{c}{ MT-TPVs versus AT-TPVs (section 3.1) } \\
\hline 1 & 2 & 3 & 4 & 5 & 6 & 7 & 8 & 9 & 10 \\
\hline 11 & 12 & 13 & 14 & 15 & 16 & 17 & 18 & 19 & 20 \\
\hline \multicolumn{7}{c}{ AT-TPVs vs. MT-TPVs (section 3.2) } \\
\hline
\end{tabular}


(a) 00 UTC 30 July 2007

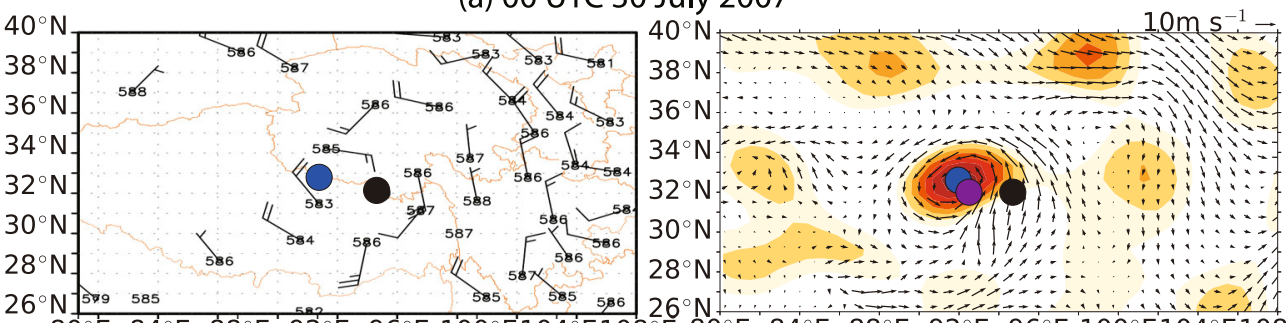

$80^{\circ} \mathrm{E} 84^{\circ} \mathrm{E} 88^{\circ} \mathrm{E} 92^{\circ} \mathrm{E} 96^{\circ} \mathrm{E} 100^{\circ} \mathrm{E} 104^{\circ} \mathrm{E} 108^{\circ} \mathrm{E} 80^{\circ} \mathrm{E} 84^{\circ} \mathrm{E} 88^{\circ} \mathrm{E} 92^{\circ} \mathrm{E} 96^{\circ} \mathrm{E} 100^{\circ} \mathrm{E} 104^{\circ} \mathrm{E} 108^{\circ} \mathrm{E}$ (b) 12 UTC 30 July 2007

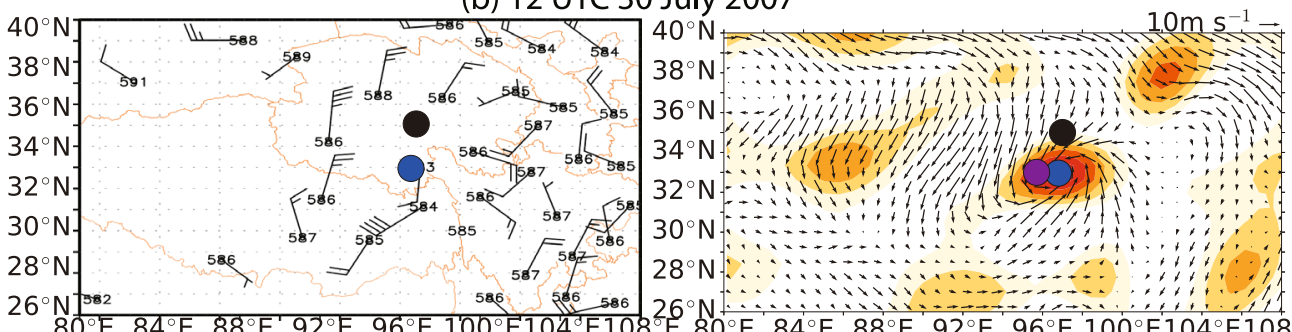

${ }_{80^{\circ} \mathrm{E}}^{\mathrm{N}} 84^{\circ} \mathrm{E} 88^{\circ} \mathrm{E} 92^{\circ} \mathrm{E} 96^{\circ} \mathrm{E} 100^{\circ} \mathrm{E} 104^{\circ} \mathrm{E} 108^{\circ} \mathrm{E} 80^{\circ} \mathrm{N} \mathrm{E} 84^{\circ} \mathrm{E} 88^{\circ} \mathrm{E} 92^{\circ} \mathrm{E} 96^{\circ} \mathrm{E} 100^{\circ} \mathrm{E} 104^{\circ} \mathrm{E} 108^{\circ} \mathrm{E}$

(c) 00 UTC 31 July 2007

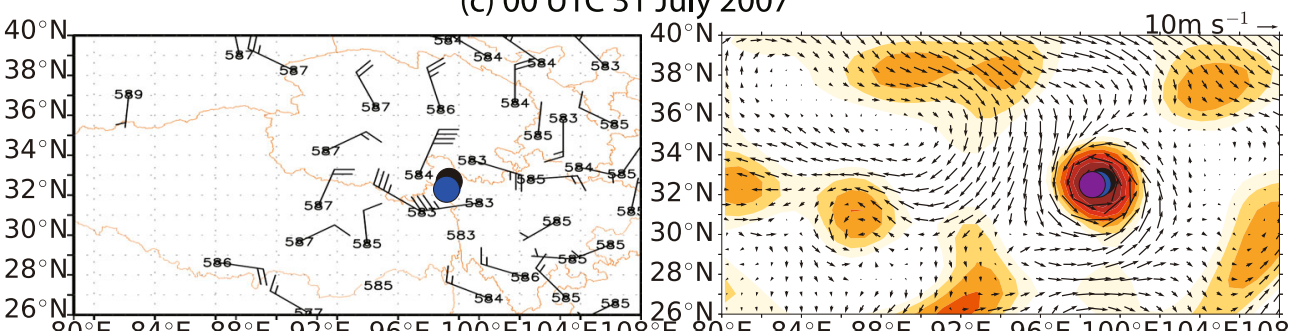

$80^{\circ} \mathrm{E} 84^{\circ} \mathrm{E} 88^{\circ} \mathrm{E} 92^{\circ} \mathrm{E} 96^{\circ} \mathrm{E} 100^{\circ} \mathrm{E} 104^{\circ} \mathrm{E} 108^{\circ} \mathrm{E} 80^{\circ} \mathrm{E} 84^{\circ} \mathrm{E} 88^{\circ} \mathrm{E} 92^{\circ} \mathrm{E} 96^{\circ} \mathrm{E} 100^{\circ} \mathrm{E} 104^{\circ} \mathrm{E} 108^{\circ} \mathrm{E}$

(d) 12 UTC 31 July 2007

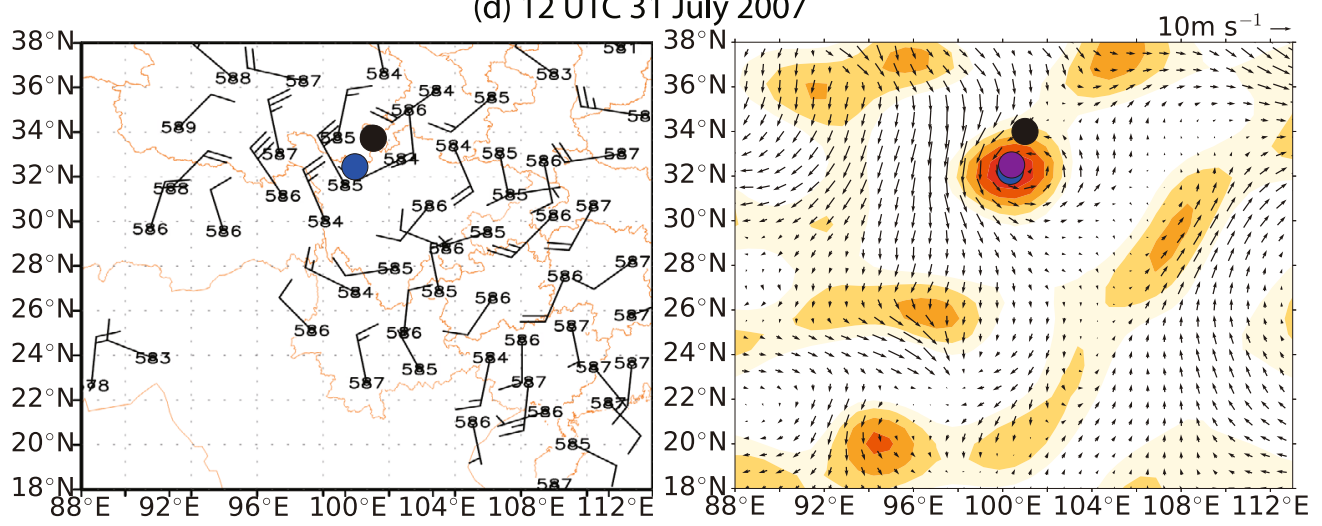

(e) 00 UTC 01 August 2007

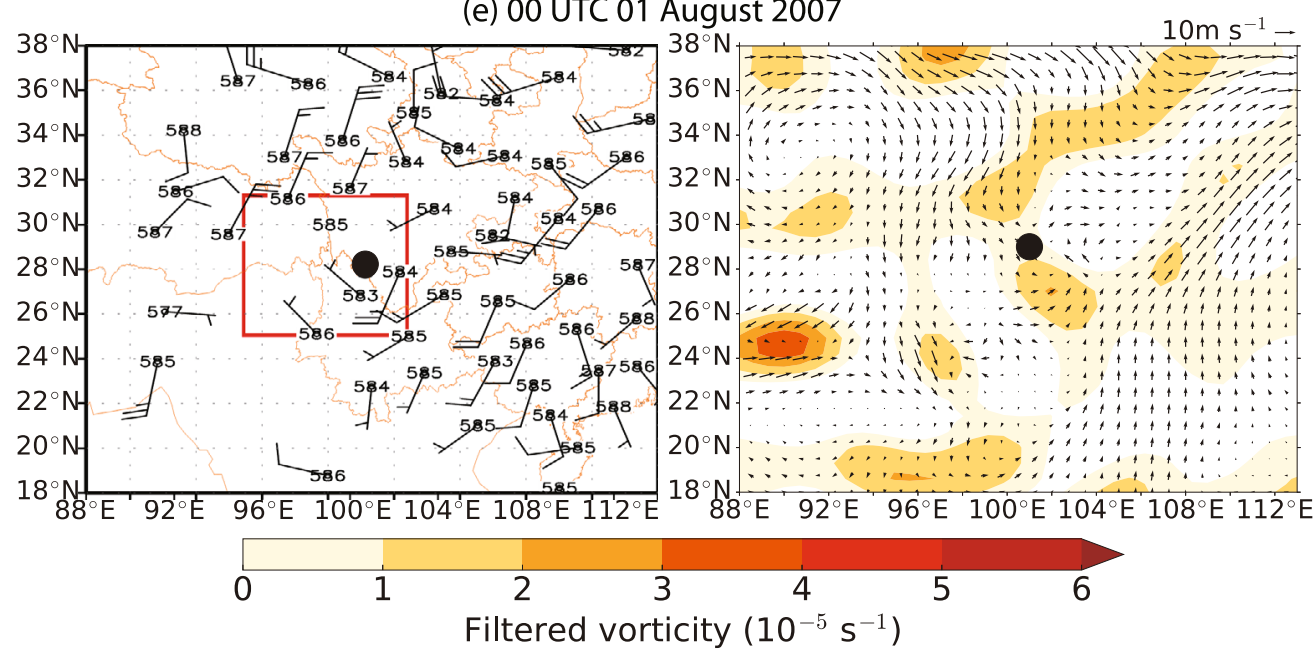

Fig. 11. As in Fig. 10 but for selected time steps (a-e) of case 9. 
TPV if it fulfils all the criteria defined in the TRACK settings (vorticity threshold, minimum persistence, geopotential minimum, originating within the TP). These settings might not yet be optimal, but could be improved using the advanced experience of experts in manual TPV detection.

The agreement of the underlying data is essential for the achievement of consistent tracking results. Large differences between the underlying data lead to different tracking results, e.g., one dataset exhibits a cyclonic circulation but the other does not.

The direction of comparison has an effect on the differences between the TPV tracks. The comparison results in a better agreement between the tracks of the partial-match TPV cases when matching MT-TPVs from the yearbooks to AT-TPVs. The MT-TPV cases found as partial matches to the selected AT-TPVs exhibit a higher number of very short tracks $(2 \times 12$-hourly time steps)—cases 3 (Fig. 6c), 5 (Fig. 6e) and 6 (Fig. 7a) — and could perhaps also be considered as non-matches. The reason for this could be the larger number of identified and tracked AT-TPVs from ERA-Interim, so that most MT-TPVs from the yearbooks are covered by the AT-TPVs from ERA-Interim, but not all AT-TPVs have a corresponding MT-TPV in the yearbooks. The best matching results, independent from the direction of comparison, are found for TPV cases exhibiting an east path.

TPV cases for which the only real difference is that the AT-TPV starts earlier and further west than the MT-TPV are still considered good matches, since the MT method cannot identify TPVs west of $90^{\circ} \mathrm{E}$ owing to the absence of sounding stations in the western part of the TP.

One of the key advantages of AT is that it is objective and reproducible, while MT performed by different experts can lead to different results. The AT approach uses a dataset that covers the entire TP and has a higher spatial and temporal resolution (6-hourly compared to 12-hourly), and could even be increased depending on the dataset used. The fact that there are no sounding stations available in the western part of the $\mathrm{TP}$, west of $90^{\circ} \mathrm{E}$, and the main genesis region for TPVs is in the west-central part of the TP, makes early detection of TPVs using MT very difficult. Therefore, the second key advantage of TRACK is that TPVs are identified further west and at an earlier stage of their lifetime, which may be helpful in a numerical-weather-prediction context. The automated TPV tracking approach could be incorporated in the operational forecasting system used by the Institute of Plateau Meteorology to improve the forecasting of TPV paths and to increase the forecasting lead time. Currently, TPVs affecting the downstream regions of the TP are often identified too late to issue appropriate warnings for, and take precautionary action against, heavy precipitation and flooding events.

The experts performing MT have the advantage of experience and in-depth knowledge of TPVs. This knowledge could be used to refine the settings of TRACK for future efforts in TPV detection and tracking to further improve automated TPV tracking.

Possible future work also includes an iteration of the current analysis using the complete set of MT-TPVs for the avail- able years (2000-14) to obtain a quantitative evaluation. This could be done using a cyclone matching technique, which has been used previously to match storm tracks, tropical easterly waves and extratropical cyclones in different reanalyses (Hodges et al., 2003, 2011).

Further research on the development, structure and behavior of TPVs and their interaction with other vortices, e.g., southwest vortices, may also lead to further improvement in the AT of TPVs in reanalysis and global climate models.

The covariability of TPVs with the Subtropical Westerly Jet and other large-scale circulation features, including the underlying mechanisms, also requires further research, which may help with assessing the predictability of TPV occurrence beyond the weather-prediction timescale.

Acknowledgements. This work and all its contributors were supported by the UK-China Research and Innovation Partnership Fund through the Met Office Climate Science for Service Partnership (CSSP) China as part of the Newton Fund grant agreement P100195 between the Met Office and the National Centre for Atmospheric Science at the University of Reading for the MESETA (Modelling Physical and Dynamical Processes over the Tibetan Plateau and their Regional Effects over East Asia) project. We thank the two anonymous reviewers for their constructive comments, which helped to improve the manuscript.

Open Access This article is distributed under the terms of the Creative Commons Attribution License which permits any use, distribution, and reproduction in any medium, provided the original author(s) and the source are credited.

\section{REFERENCES}

Bao, X. H., and F. Q. Zhang, 2013: Evaluation of NCEPCFSR, NCEP-NCAR, ERA-Interim, and ERA-40 reanalysis datasets against independent sounding observations over the Tibetan Plateau. J. Climate, 26(1), 206-214, https://doi.org/ 10.1175/JCLI-D-12-00056.1.

Bei, N. F., S. X. Zhao, and S. T. Gao, 2002: Numerical simulation of a heavy rainfall event in China during July 1998. Meteor. Atmos. Phys., 80, 153-164, https://doi.org/10.1007/ s007030200022.

Bengtsson, L., K. I. Hodges, and M. Esch, 2007: Tropical cyclones in a T159 resolution global climate model: Comparison with observations and re-analyses. Tellus A, 59(4), 396416, https://doi.org/10.1111/j.1600-0870.2007.00236.x.

Catto, J. L., L. C. Shaffrey, and K. I. Hodges, 2010: Can climate models capture the structure of extratropical cyclones? J. Climate, 23(7), 1621-1635, https://doi.org/10.1175/2009 JCLI3318.1.

Catto, J. L., L. C. Shaffrey, and K. I. Hodges, 2011: Northern hemisphere extratropical cyclones in a warming climate in the HiGEM High-Resolution climate model. J. Climate, 24(20), 5336-5352, https://doi.org/10.1175/2011JCLI4181.1.

Chen, G. X., T. Iwasaki, H. L. Qin, and W. M. Sha, 2014: Evaluation of the warm-season diurnal variability over East Asia in recent reanalyses JRA-55, ERA-Interim, NCEP CFSR, and NASA MERRA. J. Climate, 27(14), 5517-5537, https:// doi.org/10.1175/JCLI-D-14-00005.1. 
Chen, L. S., and Z. X. Luo, 2003: A preliminary study of the dynamics of eastwardshifting cyclonic vortices. Adv. Atmos. Sci., 20, 323-332, https://doi.org/10.1007/BF02690790.

Chen, Y. R., Y. Q. Li, and T. L. Zhao, 2015: Cause analysis on eastward movement of southwest China vortex and its induced heavy rainfall in South China. Advances in Meteorology, 2015, Article ID 481735, https://doi.org/10.1155/2015/ 481735.

Dee, D. P., and Coauthors, 2011: The ERA-Interim reanalysis: Configuration and performance of the data assimilation system. Quart. J. Roy. Meteor. Soc., 137, 553-597, https:// doi.org/10.1002/qj.828.

Dimri, A. P., D. Niyogi, A. P. Barros, J. Ridley, U. C. Mohanty, T. Yasunari, and D. R. Sikka, 2015: Western disturbances: A review. Rev. Geophys., 53(2), 225-246, https://doi.org/10.1002/ 2014RG000460.

Feng, X. Y., C. H. Liu, R. Rasmussen, and G. Z. Fan, 2014: A 10yr climatology of Tibetan Plateau vortices with NCEP climate forecast system reanalysis. Journal of Applied Meteorology and Climatology, 53, 34-46, https://doi.org/10.1175/JAMCD-13-014.1.

Hodges, K., A. Cobb, and P. L. Vidale, 2017: How well are tropical cyclones represented in reanalysis datasets? J. Climate, 30(14), 5243-5264, https://doi.org/10.1175/JCLI-D$16-0557.1$

Hodges, K. I., 1994: A general method for tracking analysis and its application to meteorological data. Mon. Wea. Rev., 122, 2573-2586, https://doi.org/10.1175/1520-0493(1994) 122<2573:AGMFTA > 2.0.CO;2.

Hodges, K. I., 1995: Feature tracking on the unit sphere. Mon. Wea. Rev., 123(12), 3458-3465, https://doi.org/10.1175/ 1520-0493(1995)123<3458:FTOTUS>2.0.CO;2.

Hodges, K. I., 1999: Adaptive constraints for feature tracking. Mon. Wea. Rev., 127(6), 1362-1373, https://doi.org/10.1175/ 1520-0493(1999)127<1362:ACFFT>2.0.CO;2.

Hodges, K. I., R. W. Lee, and L. Bengtsson, 2011: A comparison of extratropical cyclones in recent reanalyses ERA-Interim, NASA MERRA, NCEP CFSR, and JRA-25. J. Climate, 24, 4888-4906, https://doi.org/10.1175/2011JCLI4097.1.

Hodges, K. I., B. J. Hoskins, J. Boyle, and C. Thorncroft, 2003: A comparison of recent reanalysis datasets using objective feature tracking: Storm tracks and tropical easterly waves. Mon. Wea. Rev., 131, 2012-2037, https://doi.org/10.1175/15200493(2003)131<2012:ACORRD>2.0.CO;2.

Hoskins, B. J., and K. I. Hodges, 2002: New perspectives on the Northern hemisphere winter storm tracks. J. Atmos. Sci., 59(6), 1041-1061, https://doi.org/10.1175/1520-0469(2002) 059<1041:NPOTNH > 2.0.CO;2.

Huang, D.-Q., J. Zhu, Y.-C. Zhang, Y. Huang, and X.-Y. Kuang, 2016: Assessment of summer monsoon precipitation derived from five reanalysis datasets over East Asia. Quart. J. Roy. Meteor. Soc., 142(694), 108-119, https://doi.org/10.1002/ qj.2634.

Institute of Plateau Meteorology (IPM), and CMA, 2009: Year Book of Tibetan Plateau Vortex and Shear Line. Science Press, Beijing, 1-167. (in Chinese)

Institute of Plateau Meteorology (IPM), and CMA, 2010: Year Book of Tibetan Plateau Vortex and Shear Line. Science Press, Beijing, 1-113. (in Chinese)

Institute of Plateau Meteorology (IPM), and CMA, 2011: Year Book of Tibetan Plateau Vortex and Shear Line. Science Press, Beijing, 1-149. (in Chinese)
Institute of Plateau Meteorology (IPM), and CMA, 2012: Year Book of Tibetan Plateau Vortex and Shear Line. Science Press, Beijing, 1-158. (in Chinese)

Institute of Plateau Meteorology (IPM), and CMA, 2013: Year Book of Tibetan Plateau Vortex and Shear Line. Science Press, Beijing, 1-182. (in Chinese)

Institute of Plateau Meteorology (IPM), and CMA, 2014: Year Book of Tibetan Plateau Vortex and Shear Line. Science Press, Beijing, 1-127. (in Chinese)

Kuo, Y.-H., L. S. Cheng, and J. W. Bao, 1988: Numerical simulation of the 1981 Sichuan flood. Part I: Evolution of a mesoscale southwest Vortex. Mon. Wea. Rev., 116, 2481-2504, https://doi.org/10.1175/1520-0493(1988) 116<2481:NSOTSF $>2.0$.CO;2.

Li, G. P., and F. H. Zhao, 2017: Analysis of the mechanism underlying Tibetan Plateau vortex frequency difference between strong and weak MJO periods. J. Meteor. Res., 31(3), 530539, https://doi.org/10.1007/s13351-017-6041-6.

Li, L., R. H. Zhang, and M. Wen, 2014a: Diurnal variation in the occurrence frequency of the Tibetan Plateau vortices. Meteor. Atmos. Phys., 125, 135-144, https://doi.org/10.1007/s00703014-0325-5.

Li, L., R. H. Zhang, M. Wen, and L. K. Liu, 2014b: Effect of the atmospheric heat source on the development and eastward movement of the Tibetan Plateau vortices. Tellus A, 66(1), 24451, https://doi.org/10.3402/tellusa.v66.24451.

Li L., R. H. Zhang, and M. Wen, 2011: Diagnostic analysis of the evolution mechanism for a vortex over the Tibetan Plateau in June 2008. Adv. Atmos. Sci., 28, 797-808, https://doi.org/ 10.1007/s00376-010-0027-y.

Li, L., R. H. Zhang, and M. Wen, 2017: Genesis of southwest vortices and its relation to Tibetan Plateau vortices. Quart. J. Roy. Meteor. Soc., 143(707), 2556-2566, https://doi.org/10.1002/ qj.3106.

Li, Y. Q., and Coauthors, 2000: Year Book of Tibetan Plateau Vortex and Shear Line. Science Press, Beijing, 1-81. (in Chinese)

Li, Y. Q., and Coauthors, 2001: Year Book of Tibetan Plateau Vortex and Shear Line. Science Press, Beijing, 1-91. (in Chinese)

Li, Y. Q., and Coauthors, 2002: Year book of Tibetan Plateau Vortex and Shear Line. Science Press, Beijing, 1-100. (in Chinese)

Li, Y. Q., and Coauthors, 2003: Year Book of Tibetan Plateau Vortex and Shear Line. Science Press, Beijing, 1-100. (in Chinese)

Li, Y. Q., and Coauthors, 2004: Year Book of Tibetan Plateau Vortex and Shear Line. Science Press, Beijing, 1-116. (in Chinese)

Li, Y. Q., and Coauthors, 2005: Year Book of Tibetan Plateau Vortex and Shear Line. Science Press, Beijing, 1-145. (in Chinese)

Li, Y. Q., and Coauthors, 2006: Year Book of Tibetan Plateau Vortex and Shear Line. Science Press, Beijing, 1-128. (in Chinese)

Li, Y. Q., and Coauthors, 2007: Year Book of Tibetan Plateau Vortex and Shear Line. Science Press, Beijing, 1-145. (in Chinese)

Li, Y. Q., and Coauthors, 2008: Year Book of Tibetan Plateau Vortex and Shear Line. Science Press, Beijing, 1-140. (in Chinese)

Lin, R. P., T. J. Zhou, and Y. Qian, 2014: Evaluation of global monsoon precipitation changes based on five reanalysis datasets. J. Climate, 27(3), 1271-1289, https://doi.org/10.1175/JCLI- 
D-13-00215.1.

Lin, Z. Q., 2015: Analysis of Tibetan Plateau vortex activities using ERA-Interim data for the period 1979-2013. J. Meteor. Res., 29(5), 720-734, https://doi.org/10.1007/s13351-015-4273-x.

Noer, G., Ø. Saetra, T. Lien, and Y. Gusdal, 2011: A climatological study of polar lows in the Nordic Seas. Quart. J. Roy. Meteor. Soc., 137, 1762-1772, https://doi.org/10.1002/qj.846.

Sardeshmukh, P. D., and B. I. Hoskins, 1984: Spatial smoothing on the sphere. Mon. Wea. Rev., 112(12), 2524-2529, https://doi. org/10.1175/1520-0493(1984)112<2524:SSOTS >2.0.CO;2.

Tao, S.-Y., and Y.-H. Ding, 1981: Observational evidence of the influence of the Qinghai-Xizang (Tibet) Plateau on the occurrence of heavy rain and severe convective storms in China. Bull. Amer. Meteor. Soc., 62, 23-30, https://doi.org/10.1175/ 1520-0477(1981)062<0023:OEOTIO>2.0.CO;2.

Tous, M., and R. Romero, 2013: Meteorological environments associated with medicane development. International Journal of Climatology, 33, 1-14, https://doi.org/10.1002/joc.3428.

Tous, M., G. Zappa, R. Romero, L. Shaffrey, and P. L. Vidale, 2016: Projected changes in medicanes in the HadGEM3 N512 high-resolution global climate model. Climate Dyn., 47(5-6), 1913-1924, https://doi.org/10.1007/s00382-015-2941-2.

Wang, B., 1987: The development mechanism for Tibetan Plateau warm vortices. J. Atmos. Sci., 44, 2978-2994, https://doi.org/ 10.1175/1520-0469(1987)044<2978:TDMFTP>2.0.CO;2.

Wang, B., and I. Orlanski, 1987: Study of a heavy rain vortex formed over the eastern flank of the Tibetan Plateau. Mon. Wea. Rev., 115(7), 1370-1393, https://doi.org/10.1175/15200493(1987)115<1370:SOAHRV>2.0.CO;2.

Wu, G.-X., and S.-J. Chen, 1985: The effect of mechanical forcing on the formation of a mesoscale vortex. Quart. J. Roy. Meteor. Soc., 111, 1049-1070, https://doi.org/10.1002/ qj.49711147009.
Xiang, S. Y., Y. Q. Li, D. Li, and S. Yang, 2013: An analysis of heavy precipitation caused by a retracing plateau vortex based on TRMM data. Meteor. Atmos. Phys., 122, 33-45, https:// doi.org/10.1007/s00703-013-0269-1.

Yu, S. H., and W. L. Gao, 2009: Large-scale conditions of Tibet Plateau vortex departure. Sciences in Cold and Arid Regions, 1, 559-569.

Yu, S. H., W. L. Gao, J. Peng, and Y. H. Xiao, 2014: Observational facts of sustained departure plateau vortexes. J. Meteor. Res., 28(2), 296-307, https://doi.org/10.1007/s13351-014-3023-9.

Yu, S. H., W. L. Gao, D. X. Xiao, and J. Peng, 2016: Observational facts regarding the joint activities of the southwest vortex and plateau vortex after its departure from the Tibetan Plateau. Adv. Atmos. Sci., 33(1), 34-46, https://doi.org/ 10.1007/s00376-015-5039-1.

Zappa, G., L. Shaffrey, and K. Hodges, 2014: Can polar lows be objectively identified and tracked in the ECMWF operational analysis and the ERA-interim reanalysis? Mon. Wea. Rev., 142(8), 2596-2608, https://doi.org/10.1175/mwr-d-1400064.1.

Zappa, G., L. C. Shaffrey, K. I. Hodges, P. G. Sansom, and D. B. Stephenson, 2013: A multimodel assessment of future projections of North Atlantic and European extratropical cyclones in the CMIP5 climate models. J. Climate, 26(16), 5846-5862, https://doi.org/10.1175/JCLI-D-12-00573.1.

Zhang, P. F., G. P. Li, X. H. Fu, and Y. M. Liu, 2014: Clustering of Tibetan Plateau vortices by 10-30-Day intraseasonal oscillation. Mon. Wea. Rev., 142, 290-300, https://doi.org/10.1175/ MWR-D-13-00137.1.

Zhao, F.-H., G.-P. Li, C.-H. Huang, and X.-R. Liu, 2016: Modulation of Madden-Julian oscillation on Tibetan Plateau vortex. Journal of Tropical Meteorology, 22, 30-41, https://doi.org/ 10.16555/j.1006-8775.2016.01.004. 